\title{
Research Article \\ Simplices in the Endomorphism Semiring of a Finite Chain
}

\author{
Ivan Trendafilov \\ Department of Algebra and Geometry, Faculty of Applied Mathematics and Informatics, \\ Technical University of Sofia, Sofia, Bulgaria \\ Correspondence should be addressed to Ivan Trendafilov; ivan_d_trendafilov@tu-sofia.bg
}

Received 30 August 2013; Accepted 7 March 2014; Published 27 April 2014

Academic Editor: Andrei V. Kelarev

Copyright (C) 2014 Ivan Trendafilov. This is an open access article distributed under the Creative Commons Attribution License, which permits unrestricted use, distribution, and reproduction in any medium, provided the original work is properly cited.

We establish new results concerning endomorphisms of a finite chain, if the cardinality of the image of such endomorphism is no more than some fixed number. The semiring of all such endomorphisms can be seen as a simplex whose vertices are the constant endomorphisms. We explore the properties of these simplices.

\section{Introduction and Preliminaries}

It is well known that each simplicial complex has a geometric (continuous) interpretation as a convex set spanned by $k$ geometrically independent points in some Euclidean space.

Here, we present an algebraic (discrete) interpretation of simplicial complex as a subsemiring, containing (in some sense spanned by) $k$ constant endomorphisms of the endomorphism semiring $\widehat{\mathscr{C}}_{\mathscr{C}_{n}}$ of a finite chain. The endomorphism semiring of a finite semilattice is well studied in [1-10].

The paper is organized as follows. After the introduction and preliminaries, in Section 2, we give basic definitions and obtain some elementary properties of simplices. Although we do not speak about any distance here, we define discrete neighborhoods with respect to any vertex of the simplex. In Section 3, we study discrete neighborhoods, left ideals, and right ideals of a simplex. The main results are Theorems 9 and 12, where we find two right ideals of simplex. In Section 4, Theorem 15 is the main result of the paper, where we show that important objects (idempotents, $a$-nilpotent elements, left ideals, and right ideals) of simplex (big semiring) can be constructed using similar objects of coordinate simplex (little semiring).

Since the terminology for semirings is not completely standardized, we say what our conventions are. An algebra $R=(R,+, \cdot)$, with two binary operations + and $\cdot$ on $R$, is called a semiring if:

(i) $(R,+)$ is a commutative semigroup;

(ii) $(R, \cdot)$ is a semigroup; (iii) both distributive laws hold $x \cdot(y+z)=x \cdot y+x \cdot z$ and $(x+y) \cdot z=x \cdot z+y \cdot z$, for any $x, y, z \in R$.

Let $R=(R,+, \cdot)$ be a semiring. If a neutral element 0 of the semigroup $(R,+)$ exists and $0 x=0$ or $x 0=0$, it is called a left or a right zero, respectively, for all $x \in R$. If $0 \cdot x=x \cdot 0=0$, for all $x \in R$, then it is called zero. An element $e$ of a semigroup $(R, \cdot)$ is called a left (right) identity provided that $e x=x$ or $x e=x$, respectively, for all $x \in R$. If a neutral element 1 of the semigroup $(R, \cdot)$ exists, it is called identity.

A nonempty subset $I$ of $R$ is called an ideal if $I+I \subseteq I$, $R I \subseteq I$, and $I R \subseteq I$.

The facts concerning semirings can be found in [1].

For a join-semilattice $(\mathscr{M}, \mathrm{V})$, set $\mathscr{E}_{\mathscr{M}}$ of the endomorphisms of $\mathscr{M}$ to be a semiring with respect to the addition and multiplication defined as follows:

(i) $h=f+g$, when $h(x)=f(x) \vee g(x)$, for all $x \in \mathscr{M}$;

(ii) $h=f \cdot g$, when $h(x)=f(g(x))$, for all $x \in \mathscr{M}$.

This semiring is called the endomorphism semiring of $\mathscr{M}$.

In this paper, all semilattices are finite chains. Following [2], we fix a finite chain $\mathscr{C}_{n}=(\{0,1, \ldots, n-1\}, \vee)$ and denote the endomorphism semiring of this chain with $\widehat{\mathscr{C}}_{\mathscr{C}_{n}}$. We do not assume that $\alpha(0)=0$ for arbitrary $\alpha \in \widehat{\mathscr{C}}_{\mathscr{C}_{n}}$. So, there is not a zero in endomorphism semiring $\widehat{\mathscr{C}}_{\mathscr{C}_{n}}$. Subsemirings $\mathscr{C}_{\mathscr{C}_{n}}^{(a)}$, where $a \in \mathscr{C}_{n}$, of the semiring $\widehat{\mathscr{C}}_{\mathscr{C}_{n}}$, consisting of all endomorphisms $\alpha$ with fixed point $a$, are considered in [3].

If $\alpha \in \widehat{\mathscr{C}}_{\mathscr{C}_{n}}$ such that $f(k)=i_{k}$, for any $k \in \mathscr{C}_{n}$, we denote $\alpha$ as an ordered $n$-tuple $2 i_{0}, i_{1}, i_{2}, \ldots, i_{n-1}$. Note 
that the mappings will be composed accordingly, although we shall usually give preference to writing mappings on the right, so that $\alpha \cdot \beta$ means "first $\alpha$, then $\beta$ ". The identity $\mathbf{i}=$ $\imath 0,1, \ldots, n-1 \imath$ and all constant endomorphisms $\bar{k}=\imath k, \ldots, k \imath$ are obviously (multiplicatively) idempotents.

Let $a \in \mathscr{C}_{n}$. For every endomorphism $\bar{a}=2 a a \ldots a$, the elements of

$$
\begin{aligned}
\mathcal{N}_{n}^{[a]}=\left\{\alpha \mid \alpha \in \widehat{\mathscr{E}}_{\mathscr{C}_{n}},\right. \\
\left.\alpha^{n_{a}}=\bar{a} \text { for some natural number } n_{a}\right\}
\end{aligned}
$$

are called $a$-nilpotent endomorphisms. An important result for $a$-nilpotent endomorphisms is as follows.

Theorem 1 (see [4, Theorem 3.3]). For any natural $n, n \geq 2$, and $a \in \mathscr{C}_{n}$, the set of a-nilpotent endomorphisms $\mathcal{N}_{n}^{[a]}$ is a subsemiring of $\widehat{\mathscr{E}}_{\mathscr{C}_{n}}$. The order of this semiring is $\left|\mathcal{N}_{n}^{[k]}\right|=C_{k}$. $C_{n-k-1}$, where $C_{k}$ is the kth Catalan number.

Another useful result is as follows.

Theorem 2 (see [5, Theorem 9]). The subset of $\widehat{\mathscr{E}}_{\mathscr{C}_{n}}, n \geq 3$, of all idempotent endomorphisms with s fixed points $k_{1}, \ldots, k_{s}$, $1 \leq s \leq n-1$ is a semiring of order $\prod_{m=1}^{s-1}\left(k_{m+1}-k_{m}\right)$.

For definitions and results concerning simplices, we refer the reader to $[6,7]$.

\section{The Simplex $\sigma^{(n)}\left\{a_{0}, \ldots, a_{k-1}\right\}$}

Let us fix elements $a_{0}, \ldots, a_{k-1} \in \mathscr{C}_{n}$, where $k \leq n, a_{0}<\cdots<$ $a_{k-1}$, and let $A=\left\{a_{0}, \ldots, a_{k-1}\right\}$. We consider endomorphisms $\alpha \in \widehat{\mathscr{E}}_{\mathscr{C}_{n}}$ such that $\operatorname{Im}(\alpha) \subseteq A$. We denote this set by $\sigma^{(n)}\left\{a_{0}, \ldots, a_{k-1}\right\}$.

Let $\left\{b_{0}, \ldots, b_{\ell-1}\right\} \subseteq\left\{a_{0}, \ldots, a_{k-1}\right\}$ and consider the set

$$
\begin{array}{r}
\sigma^{(n)}\left\{b_{0}, \ldots, b_{\ell-1}\right\}=\left\{\beta \mid \beta \in \sigma^{(n)}\left\{a_{0}, \ldots, a_{k-1}\right\},\right. \\
\left.\operatorname{Im}(\beta)=\left\{b_{0}, \ldots, b_{\ell-1}\right\}\right\} .
\end{array}
$$

For $\beta_{1}, \beta_{2} \in \sigma^{(n)}\left\{b_{0}, \ldots, b_{k-1}\right\}$, let $\beta_{1} \sim \beta_{2}$, if and only if the sets $\operatorname{Im}\left(\beta_{1}\right)$ and $\operatorname{Im}\left(\beta_{2}\right)$ have a common least element. In this way, we define an equivalence relation. Any equivalence class can be identified with its least element which is the constant endomorphism $\overline{b_{m}}=2 b_{m}, \ldots, b_{m} 2$, where $m=0, \ldots l-1$.

Now take a simplicial complex $\Delta$ with vertex set $V=$ $\left\{\overline{a_{0}}, \ldots, \overline{a_{k-1}}\right\}$. The subset $\left\{\overline{b_{0}}, \ldots, \overline{b_{\ell-1}}\right\}$ is a face of $\Delta$. Hence, we can consider the set $\sigma^{(n)}\left\{b_{0}, \ldots, b_{\ell-1}\right\}$ as a face of $\Delta$. In particular, when the simplicial complex $\Delta$ consists of all subsets of $V$, it is called a simplex (see [6]) and $\Delta=$ $\sigma^{(n)}\left\{a_{0}, \ldots, a_{k-1}\right\}$.

It is easy to see that $\operatorname{Im}(\alpha) \subseteq A$ and $\operatorname{Im}(\beta) \subseteq A$ imply $\operatorname{Im}(\alpha+\beta) \subseteq A$ and $\operatorname{Im}(\alpha \cdot \beta) \subseteq A$, and so we have proved the following.
Proposition 3. For any set $A=\left\{a_{0}, \ldots, a_{k-1}\right\} \subseteq \mathscr{C}_{n}$, the simplex $\sigma^{(n)}\left\{a_{0}, \ldots, a_{k-1}\right\}$ is a subsemiring of $\widehat{\mathscr{E}}_{\mathscr{C}_{n}}$.

The number $k$ is called a dimension of simplex $\sigma^{(n)}\left\{a_{0}, \ldots, a_{k-1}\right\}$. Any simplex $\sigma^{(n)}\left\{b_{0}, b_{1}, \ldots, b_{\ell-1}\right\}$, where $b_{0}, \ldots, b_{\ell-1} \in A$, is a face of simplex $\sigma^{(n)}\left\{a_{0}, \ldots, a_{k-1}\right\}$. If $\ell<k$, face $\sigma^{(n)}\left\{b_{0}, b_{1}, \ldots, b_{\ell-1}\right\}$ is called a proper face.

The proper faces of simplex $\sigma^{(n)}\left\{a_{0}, \ldots, a_{k-1}\right\}$ are as follows:

(i) 0 -simplices are vertices $\overline{a_{0}}, \ldots, \overline{a_{k}}$;

(ii) 1-simplices are called strings; they are denoted by $\mathcal{S} \mathscr{T} \mathscr{R}^{(n)}\{a, b\}$, where $a, b \in A ;$

(iii) 2-simplices are called triangles; they are denoted by $\Delta^{(n)}\{a, b, c\}$, where $a, b, c \in A ;$

(iv) 3-simplices are called tetrahedra; they are denoted by $\mathscr{T} \mathscr{E} \mathscr{T} \mathscr{R}^{(n)}\{a, b, c, d\}$, where $a, b, c, d \in A ;$

(v) the last proper faces are simplices $\sigma_{k-1}^{(n)}\left\{b_{0}, \ldots, b_{k-2}\right\}$, where $\left\{b_{0}, \ldots, b_{k-2}\right\} \subset A$.

The boundary of the simplex $\sigma^{(n)}\left\{a_{0}, \ldots, a_{k-1}\right\}$ is a union of all its proper faces and is denoted by $\partial\left(\sigma^{(n)}\left\{a_{0}, \ldots, a_{k-1}\right\}\right)$. The set

$$
\begin{aligned}
\mathscr{I} \mathscr{N} \mathscr{T} & \left(\sigma^{(n)}\left\{a_{0}, \ldots, a_{k-1}\right\}\right) \\
= & \sigma_{k}^{(n)}(A) \backslash \partial\left(\sigma^{(n)}\left\{a_{0}, \ldots, a_{k-1}\right\}\right)
\end{aligned}
$$

is called an interior of simplex $\sigma^{(n)}\left\{a_{0}, \ldots, a_{k-1}\right\}$.

It follows that the interior of simplex $\sigma^{(n)}\left\{a_{0}, \ldots, a_{k-1}\right\}$ consists of endomorphisms $\alpha$, such that $\operatorname{Im}(\alpha)=\left\{a_{0}, a_{1}\right.$, $\left.\ldots, a_{k-1}\right\}$. So, we have the following.

Proposition 4. The interior $\mathscr{I} \mathscr{N} \mathscr{T}\left(\sigma^{(n)}\left\{a_{0}, \ldots, a_{k-1}\right\}\right)$ is an additive semigroup.

Proposition 5. Any face of simplex $\sigma^{(n)}\left\{a_{0}, \ldots, a_{k-1}\right\}$ is a left ideal.

Proof. Let $\sigma^{(n)}\left\{b_{0}, \ldots, b_{\ell-1}\right\}$ be a face of simplex $\sigma^{(n)}\left\{a_{0}, \ldots, a_{k-1}\right\}$. Obviously the face is a subsemiring of $\sigma^{(n)}\left\{a_{0}, \ldots, a_{k-1}\right\}$. Let $\alpha \in \sigma^{(n)}\left\{b_{0}, \ldots, b_{\ell-1}\right\}$ and $\beta \in \sigma^{(n)}$ $\left\{a_{0}, \ldots, a_{k-1}\right\}$. Since for any $i \in \mathscr{C}_{n}$ we have $\varphi(i) \in\left\{a_{0}\right.$, $\left.\ldots, a_{k-1}\right\}$, then $(\varphi \cdot \alpha)(i) \in\left\{b_{0}, \ldots, b_{\ell-1}\right\}$. Thus $\varphi \cdot \alpha \in \sigma^{(n)}$ $\left\{b_{0}, \ldots, b_{\ell-1}\right\}$. Hence, $\sigma^{(n)}\left\{b_{0}, \ldots, b_{\ell-1}\right\}$ is a left ideal of simplex $\sigma^{(n)}\left\{a_{0}, \ldots, a_{k-1}\right\}$.

Note that any face of some simplex is not a right ideal of the simplex. For instance, take the vertex $\overline{a_{m}} \notin$ $\sigma^{(n)}\left\{b_{0}, \ldots, b_{\ell-1}\right\}$. Then, $\overline{b_{s}} \cdot \overline{a_{m}}=\overline{a_{m}} \notin \sigma^{(n)}\left\{b_{0}, \ldots, b_{\ell-1}\right\}$, for all $s=0, \ldots, \ell-1$. From the last proposition consider the following.

Corollary 6. The boundary $\partial\left(\sigma^{(n)}\left\{a_{0}, \ldots, a_{k-1}\right\}\right)$ is a multiplicative semigroup. 
The boundary and the interior of a simplex are, in general, not semirings.

For any natural $n$, endomorphism semiring $\widehat{\mathscr{E}}_{\mathscr{C}_{n}}$ is a simplex with vertices $\overline{0}, \ldots, \overline{n-1}$. The interior of this simplex consists of endomorphisms $\alpha$, such that $\operatorname{Im}(\alpha)=\mathscr{C}_{n}$. Since the latter is valid only for identity $\mathbf{i}=20,1, \ldots, n-12$, it follows that $\mathscr{I} \mathscr{N} \mathscr{T}\left(\widehat{\mathscr{E}}_{\mathscr{C}_{n}}\right)=\mathbf{i}$.

There is a following partial ordering of the faces of dimension $k-1$ of simplex $\sigma^{(n)}\left\{a_{0}, \ldots, a_{k-1}\right\}$ : least face does not contain the vertex $\overline{a_{k-1}}$ and biggest face does not contain the vertex $\overline{a_{0}}$.

The biggest face of the simplex $\widehat{\mathscr{E}}_{\mathscr{C}_{n}}$ is the simplex $\sigma^{(n)}\{1, \ldots, n-1\}$. Now $\widehat{\mathscr{E}}_{\mathscr{C}_{n}} \backslash \sigma^{(n)}\{1, \ldots, n-1\}=\mathscr{E}_{\mathscr{C}_{n}}^{(0)}$ which is a subsemiring of $\widehat{\mathscr{E}}_{\mathscr{C}_{n}}$. Similarly, the least face of $\widehat{\mathscr{C}}_{\mathscr{C}_{n}}$ is $\sigma^{(n)}\{0, \ldots, n-2\}$. Then, $\widehat{\mathscr{E}}_{\mathscr{C}_{n}} \backslash \sigma^{(n)}\{0, \ldots, n-2\}=\mathscr{E}_{\mathscr{C}_{n}}^{(n-1)}$ which is also a subsemiring of $\widehat{\mathscr{E}}_{\mathscr{C}_{n}}$. The other faces of $\widehat{\mathscr{C}}_{\mathscr{C}_{n}}$, where $n \geq 3$, do not have this property. Indeed, one middle face is $\sigma^{(n)}\{0, \ldots, k-1, k+1, \ldots, n-1\}$. But set $R=\widehat{\mathscr{E}}_{\mathscr{C}_{n}} \backslash$ $\sigma^{(n)}\{0, \ldots, k-1, k+1, \ldots, n-1\}$ is not a semiring because, for any $n \geq 3$ and any $k \in\{1, \ldots, n-2\}$, if $\alpha=\imath 0, \ldots, 0, k \imath \in R$, then $\alpha^{2}=\overline{0} \notin R$.

Let us fix vertex $\overline{a_{m}}$, where $m=0, \ldots, k-1$ of simplex $\sigma^{(n)}\left\{a_{0}, \ldots, a_{k-1}\right\}$. The set of all endomorphisms $\alpha \in \sigma^{(n)}\left\{a_{0}, \ldots, a_{k-1}\right\}$ such that $\alpha(i)=a_{m}$ just for $s$ elements $i \in \mathscr{C}_{n}$ is called sth layer of the simplex with respect to $\overline{a_{m}}$, where $s=0, \ldots, n-1$. We denote the $s$ th layer of the simplex $\sigma^{(n)}\left\{a_{0}, \ldots, a_{k-1}\right\}$ with respect to $\overline{a_{m}}$ by $\mathscr{L}_{a_{m}}^{s}\left(\sigma^{(n)}\left\{a_{0}, \ldots, a_{k-1}\right\}\right)$. So, the 0 -layer with respect to any vertex of the simplex $\sigma^{(n)}\left\{a_{0}, \ldots, a_{k-1}\right\}$ is a face of the simplex; hence, it is a semiring. In the general case, the $s$ th layer $\mathscr{L}_{a_{m}}^{s}\left(\sigma^{(n)}\left\{a_{0}, \ldots, a_{k-1}\right\}\right)$, where $s \in \mathscr{C}_{n}, s=1, \ldots, n-2$, is not a subsemiring of simplex $\sigma^{(n)}\left\{a_{0}, \ldots, a_{k-1}\right\}$.

On the other hand, since $\mathscr{L}_{a_{m}}^{s}\left(\sigma^{(n)}\left\{a_{0}, \ldots, a_{k-1}\right\}\right)$ consists of all endomorphisms $\alpha$, such that $\alpha(i)=a_{m}$ just for $s$ elements $i \in \mathscr{C}_{n}$, it follows that this sth layer is closed under the addition. Hence, we have the following.

Proposition 7. Any layer $\mathscr{L}_{a_{m}}^{s}\left(\sigma^{(n)}\left\{a_{0}, \ldots, a_{k-1}\right\}\right)$ of simplex $\sigma^{(n)}\left\{a_{0}, \ldots, a_{k-1}\right\}$ is an additive semigroup.

Let $\overline{a_{m}}$ be an arbitrary vertex of simplex $\sigma^{(n)}\left\{a_{0}, \ldots, a_{k-1}\right\}$. From a topological point of view, the set $\mathscr{D} \mathcal{N}_{m}^{1}=\left\{\overline{a_{m}}\right\} \cup$ $\mathscr{L}_{a_{m}}^{n-1}\left(\sigma^{(n)}\left\{a_{0}, a_{1}, \ldots, a_{k-1}\right\}\right)$ is a discrete neighborhood consisting of the "nearest points to point $\overline{a_{m}}$." Similarly, we define $\mathscr{D} \mathcal{N}_{m}^{2}=\mathscr{D} \mathscr{N}_{m}^{1} \cup \mathscr{L}_{a_{m}}^{n-2}\left(\sigma^{(n)}\left\{a_{0}, a_{1}, \ldots, a_{k-1}\right\}\right)$. More generally

$$
\mathscr{D} \mathscr{N}_{m}^{t}=\left\{\overline{a_{m}}\right\} \cup \bigcup_{\ell=n-t}^{n-1} \mathscr{L}_{a_{m}}^{\ell}\left(\sigma^{(n)}\left\{a_{0}, a_{1}, \ldots, a_{k-1}\right\}\right),
$$

where $m=0, \ldots, k-1$ and $t=1, \ldots, n$ is called discrete $t$ neighborhood of the vertex $\overline{a_{m}}$.

\section{Subsemirings and Ideals of the Simplex}

$$
\sigma^{(n)}\left\{a_{0}, \ldots, a_{k-1}\right\}
$$

Lemma 8. Let $\overline{a_{m}}$, where $m=0, \ldots, k-1$, be a vertex of the simplex $\sigma^{(n)}\left\{a_{0}, a_{1}, \ldots, a_{k-1}\right\}$ and $\mathscr{L}_{a_{m}}^{n-1}\left(\sigma^{(n)}\left\{a_{0}, a_{1}, \ldots, a_{k-1}\right\}\right)$ be the $(n-1)$ th layer of the $k$-simplex with respect to $\overline{a_{m}}$. Then, the set $\mathscr{D} \mathscr{N}_{m}^{1}=\left\{\overline{a_{m}}\right\} \cup \mathscr{L}_{a_{m}}^{n-1}\left(\sigma^{(n)}\left\{a_{0}, a_{1}, \ldots, a_{k-1}\right\}\right)$, where $m=0, \ldots, k-1$, is a subsemiring of $\sigma^{(n)}\left\{a_{0}, a_{1}, \ldots, a_{k-1}\right\}$.

Proof. We consider three cases.

Case 1. Let $m=0$. Then, elements of $\mathscr{D} \mathscr{N}_{0}^{1}$ are endomorphisms:

$$
\begin{aligned}
\overline{a_{0}},\left(a_{0}\right)_{n-1} a_{1} & =2 \underbrace{a_{0}, \ldots, a_{0}}_{n-1}, a_{1} 2, \ldots,\left(a_{0}\right)_{n-1} a_{k-1} \\
& =2 \underbrace{a_{0}, \ldots, a_{0}}_{n-1}, a_{k-1} 2 .
\end{aligned}
$$

Since $\overline{a_{0}}<\left(a_{0}\right)_{n-1} a_{1}<\cdots<\left(a_{0}\right)_{n-1} a_{k-1}$, it follows that set $\mathscr{D} \mathcal{N}_{0}^{1}$ is closed under the addition.

We find $\left(a_{0}\right)_{n-1} a_{i} \cdot \overline{a_{0}}=\overline{a_{0}} \cdot\left(a_{0}\right)_{n-1} a_{i}=\overline{a_{0}}$, for all $i=$ $1, \ldots, k-1$. Also we have $\left(a_{0}\right)_{n-1} a_{i} \cdot\left(a_{0}\right)_{n-1} a_{j}=\left(a_{0}\right)_{n-1} a_{j}$. $\left(a_{0}\right)_{n-1} a_{i}=\overline{a_{0}}$, for all $i, j \in\{1, \ldots, k-1\}$, with the only exception when $a_{k-1}=n-1$. Now $\left(\left(a_{0}\right)_{n-1}(n-1)\right)^{2}=$ $\left(a_{0}\right)_{n-1}(n-1),\left(a_{0}\right)_{n-1}(n-1) \cdot\left(a_{0}\right)_{n-1} a_{i}=\left(a_{0}\right)_{n-1} a_{i}$, and $\left(a_{0}\right)_{n-1} a_{i} \cdot\left(a_{0}\right)_{n-1}(n-1)=\overline{a_{0}}$. Hence, $\mathscr{D} \mathcal{N}_{0}^{1}$ is a semiring.

Case 2. Let $m=k-1$. Then, elements of $\mathscr{D} \mathcal{N}_{k-1}^{1}$ are endomorphisms:

$$
\begin{aligned}
a_{0}\left(a_{k-1}\right)_{n-1} & =2 a_{0}, \underbrace{a_{k-1}, \ldots, a_{k-1}}_{n-1} 2, \ldots, a_{k-2}\left(a_{k-1}\right)_{n-1} \\
& =2 a_{k-2}, \underbrace{a_{k-1}, \ldots, a_{k-1}}_{n-1} 2, \overline{a_{k-1}} .
\end{aligned}
$$

Since $a_{0}\left(a_{k-1}\right)_{n-1}<\cdots<a_{k-2}\left(a_{k-1}\right)_{n-1}<\overline{a_{k-1}}$, it follows that the set $\mathscr{D} \mathcal{N}_{k-1}^{1}$ is closed under the addition.

We find $a_{i}\left(a_{k-1}\right)_{n-1} \cdot \overline{a_{k-1}}=\overline{a_{k-1}} \cdot a_{i}\left(a_{k-1}\right)_{n-1}=$ $\overline{a_{k-1}}$, for all $i=1, \ldots, k-1$. Also we have $a_{i}\left(a_{k-1}\right)_{n-1}$. $a_{j}\left(a_{k-1}\right)_{n-1}=a_{j}\left(a_{k-1}\right)_{n-1} \cdot a_{i}\left(a_{k-1}\right)_{n-1}=\overline{a_{k-1}}$, for all $i, j \in$ $\{0, \ldots, k-2\}$, with also the only exception when $a_{0}=0$. We have $\left(0\left(a_{k-1}\right)_{n-1}\right)^{2}=0\left(a_{k-1}\right)_{n-1}, 0\left(a_{k-1}\right)_{n-1} \cdot a_{i}\left(a_{k-1}\right)_{n-1}=$ $a_{i}\left(a_{k-1}\right)_{n-1}$, and $a_{i}\left(a_{k-1}\right)_{n-1} \cdot 0\left(a_{k-1}\right)_{n-1}=\overline{a_{k-1}}$. Hence, $\mathscr{D} \mathcal{N}_{k-1}^{1}$ is a semiring.

Case 3. Let $0<m<k-1$. Then elements of $\mathscr{D} \mathcal{N}_{m}^{1}$ are endomorphisms:

$$
\begin{aligned}
a_{0}\left(a_{m}\right)_{n-1} & =2 a_{0}, \underbrace{a_{m}, \ldots, a_{m}}_{n-1} 2, \ldots, a_{m-1}\left(a_{m}\right)_{n-1} \\
& =2 a_{m-1}, \underbrace{a_{m}, \ldots, a_{m}}_{n-1} 2, \overline{a_{m}}, \\
\left(a_{m}\right)_{n-1} a_{m+1} & =2 \underbrace{a_{m}, \ldots, a_{m}}_{n-1}, a_{m+1} 2, \ldots,\left(a_{m}\right)_{n-1} a_{k-1} \\
& =2 \underbrace{a_{m}, \ldots, a_{m}}_{n-1}, a_{k-1} 2 .
\end{aligned}
$$


Since $a_{0}\left(a_{m}\right)_{n-1}<\cdots<a_{m-1}\left(a_{m}\right)_{n-1}<\overline{a_{m}}<\left(a_{m}\right)_{n-1} a_{m+1}<$ $\cdots<\left(a_{m}\right)_{n-1} a_{k-1}$, it follows that set $\mathscr{D} \mathcal{N}_{m}^{1}$ is closed under the addition.

Now there are four possibilities.

(1) Let $0<a_{0}$ and $a_{k-1}<n-1$. Then

$$
\begin{array}{r}
a_{i}\left(a_{m}\right)_{n-1} \cdot a_{j}\left(a_{m}\right)_{n-1}=a_{j}\left(a_{m}\right)_{n-1} \cdot a_{i}\left(a_{m}\right)_{n-1}=\overline{a_{m}} \\
\text { for any } i, j=0, \ldots, m-1, \\
\left(a_{m}\right)_{n-1} a_{i} \cdot\left(a_{m}\right)_{n-1} a_{j}=\left(a_{m}\right)_{n-1} a_{j} \cdot\left(a_{m}\right)_{n-1} a_{i}=\overline{a_{m}}
\end{array}
$$$$
\text { for any } i, j=m+1, \ldots, k-1 \text {, }
$$

$$
a_{i}\left(a_{m}\right)_{n-1} \cdot\left(a_{m}\right)_{n-1} a_{j}=\left(a_{m}\right)_{n-1} a_{j} \cdot a_{i}\left(a_{m}\right)_{n-1}=\overline{a_{m}}
$$

for any $i=0, \ldots, m-1, j=m+1, \ldots, k-1$.

Since $a_{i}\left(a_{m}\right)_{n-1} \cdot \overline{a_{m}}=\overline{a_{m}} \cdot a_{i}\left(a_{m}\right)_{n-1}=\overline{a_{m}}$, for $i=1, \ldots, m-$ 1 , and, in a similar way, $\left(a_{m}\right)_{n-1} a_{j} \cdot \overline{a_{m}}=\overline{a_{m}} \cdot\left(a_{m}\right)_{n-1} a_{j}=\overline{a_{m}}$, for $j=m+1, \ldots, k-1$, and also $\left(\overline{a_{m}}\right)^{2}=\overline{a_{m}}$, it follows that $\mathscr{D} \mathcal{N}_{m}^{1}$ is a commutative semiring.

(2) Let $a_{0}=0$ and $a_{k-1}<n-1$. Then, $\left(0\left(a_{m}\right)_{n-1}\right)^{2}=$ $0\left(a_{m}\right)_{n-1}$,

$$
\begin{gathered}
0\left(a_{m}\right)_{n-1} \cdot a_{i}\left(a_{m}\right)_{n-1}=a_{i}\left(a_{m}\right)_{n-1}, \\
a_{i}\left(a_{m}\right)_{n-1} \cdot 0\left(a_{m}\right)_{n-1}=\overline{a_{m}} \\
\text { for any } i=1, \ldots, m-1, \\
0\left(a_{m}\right)_{n-1} \cdot\left(a_{m}\right)_{n-1} a_{j}=\left(a_{m}\right)_{n-1} a_{j} \cdot 0\left(a_{m}\right)_{n-1}=\overline{a_{m}} \\
\text { for any } j=m+1, \ldots, k-1 .
\end{gathered}
$$

We also observe that $\overline{a_{m}} \cdot 0\left(a_{m}\right)_{n-1}=0\left(a_{m}\right)_{n-1} \cdot \overline{a_{m}}=\overline{a_{m}}$. All the other equalities between the products of the elements of $\mathscr{D} \mathscr{N}_{m}^{1}$ are the same as in (1).

(3) Let $a_{0}>0$ and $a_{k-1}=n-1$. Then, $\left(\left(a_{m}\right)_{n-1}(n-1)\right)^{2}=$ $\left(a_{m}\right)_{n-1}(n-1)$,

$$
\begin{aligned}
& \left(a_{m}\right)_{n-1}(n-1) \cdot a_{i}\left(a_{m}\right)_{n-1} \\
& =a_{i}\left(a_{m}\right)_{n-1} \cdot\left(a_{m}\right)_{n-1}(n-1)=\overline{a_{m}} \\
& \text { for any } i=1, \ldots m-1, \\
& \left(a_{m}\right)_{n-1}(n-1) \cdot\left(a_{m}\right)_{n-1} a_{j}=\left(a_{m}\right)_{n-1} a_{j}, \\
& \left(a_{m}\right)_{n-1} a_{j} \cdot\left(a_{m}\right)_{n-1}(n-1)=\overline{a_{m}} \\
& \quad \text { for any } j=m+1, \ldots, k-1 .
\end{aligned}
$$

We also observe that $\overline{a_{m}} \cdot\left(a_{m}\right)_{n-1}(n-1)=\left(a_{m}\right)_{n-1}(n-1)$. $\overline{a_{m}}=\overline{a_{m}}$. All the other equalities between the products of the elements of $\mathscr{D} \mathcal{N}_{m}^{1}$ are the same as in (1).

(4) Let $a_{0}=0$ and $a_{k-1}=n-1$. Now all equalities between the products of the elements of $\mathscr{D} \mathcal{N}_{m}^{1}$ are the same as in (1), (2), and (3). So, $\mathscr{D} \mathcal{N}_{m}^{1}$ is a semiring.

Theorem 9. The union $J=\bigcup_{m=0}^{k-1} \mathscr{D} \mathcal{N}_{m}^{1}$ of the discrete 1-neighborhoods with respect to all vertices of the simplex $\sigma^{(n)}\left\{a_{0}, \ldots, a_{k-1}\right\}$ is a right ideal of the simplex.
Proof. Let $\alpha \in \mathscr{D} \mathcal{N}_{m}^{1}$. Then, $\alpha=\overline{a_{m}}, \alpha=a_{i}\left(a_{m}\right)_{n-1}$, or $\alpha=$ $\left(a_{m}\right)_{n-1} a_{j}$, where $i=0, \ldots, m-1$ and $j=m+1, \ldots, n-1$. Let $\beta \in \mathscr{D} \mathcal{N}_{s}^{1}$. Then $\beta=\overline{a_{s}}, \beta=a_{p}\left(a_{s}\right)_{n-1}$, or $\beta=\left(a_{s}\right)_{n-1} a_{q}$, where $p=0, \ldots, s-1$ and $q=s+1, \ldots, n-1$.

Suppose that $s>m$. Then, we find $\alpha+\beta=\overline{a_{s}}$ or $\alpha+\beta=$ $\left(a_{s}\right)_{n-1} a_{t}$, where $t=\max (j, q)$. So, in all cases $\alpha+\beta \in J$, what means that $J$ is closed under the addition.

Let $\alpha \in J$ and $\varphi \in \sigma^{(n)}\left\{a_{0}, \ldots, a_{k-1}\right\}$. Then, $\alpha \in \mathscr{D} \mathcal{N}_{m}^{1}$, for some $m=0, \ldots, k-1$.

If $\alpha=\overline{a_{m}}$ and $\varphi\left(a_{m}\right)=a_{s}$, then it follows that $\alpha \cdot \varphi=\overline{a_{s}} \in J$.

If $\alpha=a_{i}\left(a_{m}\right)_{n-1}$, where $i=0, \ldots, m-1, \varphi\left(a_{m}\right)=a_{s}$, and $\varphi\left(a_{i}\right)=a_{p}$, then $\alpha \cdot \varphi=a_{p}\left(a_{s}\right)_{n-1} \in J$.

If $\alpha=\left(a_{m}\right)_{n-1} a_{j}$, where $j=m+1, \ldots, k-1, \varphi\left(a_{m}\right)=a_{s}$, and $\varphi\left(a_{j}\right)=a_{q}$, it follows that $\alpha \cdot \varphi=\left(a_{s}\right)_{n-1} a_{q} \in J$.

Hence, in all cases $\alpha \cdot \varphi \in J$.

Any simplex $\sigma^{(n)}\left\{b_{0}, \ldots, b_{\ell-1}\right\}$ which is a face of simplex $\sigma^{(n)}\left\{a_{0}, \ldots, a_{k-1}\right\}$ is called internal of the simplex $\sigma^{(n)}\left\{a_{0}, \ldots, a_{k-1}\right\}$, if $a_{0} \notin \sigma^{(n)}\left\{b_{0}, \ldots, b_{\ell-1}\right\}$ and $a_{k-1} \notin$ $\sigma^{(n)}\left\{b_{0}, \ldots, b_{\ell-1}\right\}$.

Similarly simplex $\sigma^{(n)}\left\{a_{0}, \ldots, a_{k-1}\right\}$, which is a face of $n$-simplex $\widehat{\mathscr{E}}_{\mathscr{C}_{n}}$, is called internal simplex, if $0 \notin$ $\sigma^{(n)}\left\{a_{0}, \ldots, a_{k-1}\right\}$ and $n-1 \notin \sigma^{(n)}\left\{a_{0}, \ldots, a_{k-1}\right\}$.

Immediately from the proof of Proposition 4 consider the following.

Corollary 10. For any internal simplex $\sigma^{(n)}\left\{a_{0}, a_{1}, \ldots, a_{k-1}\right\}$, semirings $\mathscr{D} \mathscr{N}_{m}^{1}$ are commutative and all their elements are $a_{m}$-nilpotent, where $m=0, \ldots, k-1$.

Lemma 11. Let $\overline{a_{m}}$, where $m=0, \ldots, k-1$, be a vertex of internal simplex $\sigma^{(n)}\left\{a_{0}, \ldots, a_{k-1}\right\}$. Then, the set $\mathscr{D} \mathcal{N}_{m}^{2}=$ $\mathscr{D} \mathcal{N}_{m}^{1} \cup \mathscr{L}_{a_{m}}^{n-2}\left(\sigma^{(n)}\left\{a_{0}, \ldots, a_{k-1}\right\}\right)$, where $m=0, \ldots, k-1$, is a subsemiring of $\sigma^{(n)}\left\{a_{0}, \ldots, a_{k-1}\right\}$.

Proof. Since $\mathscr{D} \mathscr{N}_{m}^{2}=\mathscr{D} \mathscr{N}_{m}^{1} \cup \mathscr{L}_{a_{m}}^{n-2}\left(\sigma^{(n)}\left\{a_{0}, \ldots, a_{k-1}\right\}\right)$, it follows that the elements of $\mathscr{D} \mathscr{N}_{m}^{2}$ are endomorphisms:

$$
\begin{aligned}
& \overline{a_{m}} \\
& a_{i}\left(a_{m}\right)_{n-1}, \quad \text { where } i=0, \ldots, m-1, \\
& \left(a_{m}\right)_{n-1} a_{j}, \quad \text { where } j=m+1, \ldots, k-1, \\
& a_{p} a_{q}\left(a_{m}\right)_{n-2}, \quad \text { where } p, q=0, \ldots, m-1, p \leq q, \\
& \left(a_{m}\right)_{n-2} a_{r} a_{s}, \quad \text { where } r, s=m+1, \ldots, k-1, r \leq s, \\
& a_{p}\left(a_{m}\right)_{n-2} a_{s}, \quad \text { where } p=0, \ldots, m-1, \\
& \quad s=m+1, \ldots, k-1 .
\end{aligned}
$$

From Lemma 8, we know that the discrete 1neighborhood $\mathscr{D} \mathcal{N}_{m}^{1}$ is closed under the addition. From Proposition 7, it follows that the layer $\mathscr{L}_{a_{m}}^{n-2}\left(\sigma^{(n)}\left\{a_{0}, \ldots\right.\right.$, $\left.\left.a_{k-1}\right\}\right)$ also is closed under the addition. Hence, in order to 
prove that $\mathscr{D} \mathscr{N}_{m}^{2}$ is closed under the addition, we calculate the following:

$$
\begin{gathered}
a_{i}\left(a_{m}\right)_{n-1}+a_{p} a_{q}\left(a_{m}\right)_{n-2}= \begin{cases}a_{p} a_{q}\left(a_{m}\right)_{n-2}, & \text { if } i \leq p, \\
a_{i} a_{q}\left(a_{m}\right)_{n-2}, & \text { if } i>p,\end{cases} \\
a_{i}\left(a_{m}\right)_{n-1}+\left(a_{m}\right)_{n-2} a_{r} a_{s}=\left(a_{m}\right)_{n-2} a_{r} a_{s},
\end{gathered} \begin{gathered}
\left(a_{m}\right)_{n-1} a_{j}+\left(a_{m}\right)_{n-2} a_{r} a_{s}= \begin{cases}\left(a_{m}\right)_{n-2} a_{r} a_{s}, & \text { if } j \leq s, \\
\left(a_{m}\right)_{n-2} a_{r} a_{j}, & \text { if } j>s,\end{cases} \\
\left(a_{m}\right)_{n-1} a_{j}+a_{p} a_{q}\left(a_{m}\right)_{n-2}=\left(a_{m}\right)_{n-1} a_{j}, \\
a_{i}\left(a_{m}\right)_{n-1}+a_{p}\left(a_{m}\right)_{n-2} a_{s}= \begin{cases}a_{p}\left(a_{m}\right)_{n-2} a_{s}, & \text { if } i \leq p, \\
a_{i}\left(a_{m}\right)_{n-2} a_{s}, & \text { if } i>p,\end{cases} \\
\left(a_{m}\right)_{n-1} a_{j}+a_{p}\left(a_{m}\right)_{n-2} a_{s}= \begin{cases}a_{p}\left(a_{m}\right)_{n-2} a_{s}, & \text { if } j \leq s, \\
a_{p}\left(a_{m}\right)_{n-2} a_{j}, & \text { if } j>s,\end{cases} \\
\overline{a_{m}}+a_{p} a_{q}\left(a_{m}\right)_{n-2}=\overline{a_{m}}, \\
\overline{a_{m}}+\left(a_{m}\right)_{n-2} a_{r} a_{s}=\left(a_{m}\right)_{n-2} a_{r} a_{s},
\end{gathered}
$$

where $i, p, q=0,1, \ldots, m-1, p \leq q, j, r, s=m+1, \ldots, k-$ 1 , and $r \leq s$. So, we prove that the discrete 2 -neighborhood $\mathscr{D} \mathcal{N}_{m}^{2}$ is closed under the addition.

Now we consider six cases, where, for the indices, the upper restrictions are fulfilled.

Case 1. Let $a_{m}=1$. We shall show that all endomorphisms of $\mathscr{D} \mathcal{N}_{1}^{2}$ are 1-nilpotent with the only exception when $a_{k-1}=$ $n-2$. When $a_{k-1}<n-2$, since 1 is the least image of any endomorphism, there are only a few equalities: $1_{n-2} a_{r} a_{s}$. $1_{n-2} a_{r_{0}} a_{s_{0}}=\overline{1}$,

$$
\begin{gathered}
1_{n-1} a_{j} \cdot 1_{n-2} a_{r} a_{s}=1_{n-2} a_{r} a_{s} \cdot 1_{n-1} a_{j}=\overline{1}, \\
\overline{1} \cdot 1_{n-2} a_{r} a_{s}=1_{n-2} a_{r} a_{s} \cdot \overline{1}=\overline{1} .
\end{gathered}
$$

Hence, it follows that $\mathscr{D} \mathscr{N}_{1}^{2}$ is a commutative semiring with trivial multiplication.

If $a_{k-1}=n-2$, it is easy to see that endomorphism $1_{n-2}(n-2)_{2}$ is the unique idempotent of $\mathscr{D} \mathcal{N}_{1}^{2}$ (see [5]). Now, we find $1_{n-2}(n-2)_{2} \cdot 1_{n-2} a_{r} a_{s}=1_{n-2}\left(a_{r}\right)_{2}, 1_{n-1}(n-2)$. $1_{n-2} a_{r} a_{s}=1_{n-1} a_{r}$, and $1_{n-2} a_{r} a_{s} \cdot 1_{n-1}(n-2)=\overline{1}$. Hence, $\mathscr{D} \mathscr{N}_{1}^{2}$ is a semiring.

Case 2. Let $a_{m}=n-2$. We shall show that all the endomorphisms of $\mathscr{D} \mathscr{N}_{n-2}^{2}$ are 1-nilpotent with the only exception when $a_{0}=1$. When $a_{0}>1$, we find

$$
\begin{gathered}
a_{p} a_{q}(n-2)_{n-2} \cdot a_{p_{0}} a_{q_{0}}(n-2)_{n-2}=\overline{n-2}, \\
a_{i}(n-2)_{n-1} \cdot a_{p} a_{q}(n-2)_{n-2} \\
=a_{p} a_{q}(n-2)_{n-2} \cdot a_{i}(n-2)_{n-1}=\overline{n-2}, \\
\overline{n-2} \cdot a_{p} a_{q}(n-2)_{n-2}=a_{p} a_{q}(n-2)_{n-2} \cdot \overline{n-2}=\overline{n-2} .
\end{gathered}
$$

If $a_{0}=1$, the only idempotent is $1_{2}(n-2)_{n-2}$ and we find

$$
\begin{gathered}
1_{2}(n-2)_{n-2} \cdot a_{p} a_{q}(n-2)_{n-2}=\left(a_{q}\right)_{2}(n-2)_{n-2}, \\
1(n-2)_{n-1} \cdot a_{p} a_{q}(n-2)_{n-2}=a_{q}(n-2)_{n-1}, \\
a_{p} a_{q}(n-2)_{n-2} \cdot 1(n-2)_{n-1}=\overline{n-2} .
\end{gathered}
$$

Hence, $\mathscr{D} \mathcal{N}_{n-2}^{2}$ is a semiring.

Case 3. Let $1<a_{0}$ and $a_{k-1}<n-2$. We find the following trivial equalities, which are grouped by duality:

$$
\begin{aligned}
& a_{p} a_{q}\left(a_{m}\right)_{n-2} \times a_{p_{0}} a_{q_{0}}\left(a_{m}\right)_{n-2}=\overline{a_{m}}, \\
& \left(a_{m}\right)_{n-2} a_{r} a_{s} \cdot\left(a_{m}\right)_{n-2} a_{r_{0}} a_{s_{0}}=\overline{a_{m}}, \\
& a_{p} a_{q}\left(a_{m}\right)_{n-2} \cdot a_{p_{0}}\left(a_{m}\right)_{n-2} a_{s_{0}} \\
& =a_{p_{0}}\left(a_{m}\right)_{n-2} a_{s_{0}} \cdot a_{p} a_{q}\left(a_{m}\right)_{n-2}=\overline{a_{m}}, \\
& \left(a_{m}\right)_{n-2} a_{r} a_{s} \cdot a_{p_{0}}\left(a_{m}\right)_{n-2} a_{s_{0}} \\
& =a_{p_{0}}\left(a_{m}\right)_{n-2} a_{s_{0}} \cdot\left(a_{m}\right)_{n-2} a_{r} a_{s}=\overline{a_{m}}, \\
& a_{p} a_{q}\left(a_{m}\right)_{n-2} \cdot\left(a_{m}\right)_{n-2} a_{r} a_{s} \\
& =\left(a_{m}\right)_{n-2} a_{r} a_{s} \cdot a_{p} a_{q}\left(a_{m}\right)_{n-2}=\overline{a_{m}}, \\
& a_{i}\left(a_{m}\right)_{n-1} \cdot a_{p} a_{q}\left(a_{m}\right)_{n-2} \\
& =a_{p} a_{q}\left(a_{m}\right)_{n-2} \cdot a_{i}\left(a_{m}\right)_{n-1}=\overline{a_{m}}, \\
& \left(a_{m}\right)_{n-1} a_{j} \cdot a_{p} a_{q}\left(a_{m}\right)_{n-2} \\
& =a_{p} a_{q}\left(a_{m}\right)_{n-2} \cdot\left(a_{m}\right)_{n-1} a_{j}=\overline{a_{m}}, \\
& a_{i}\left(a_{m}\right)_{n-1} \cdot a_{p}\left(a_{m}\right)_{n-2} a_{s} \\
& =a_{p}\left(a_{m}\right)_{n-2} a_{s} \cdot a_{i}\left(a_{m}\right)_{n-1}=\overline{a_{m}}, \\
& \left(a_{m}\right)_{n-1} a_{j} \cdot a_{p}\left(a_{m}\right)_{n-2} a_{s} \\
& =a_{p}\left(a_{m}\right)_{n-2} a_{s} \cdot\left(a_{m}\right)_{n-1} a_{j}=\overline{a_{m}}, \\
& a_{i}\left(a_{m}\right)_{n-1} \cdot\left(a_{m}\right)_{n-2} a_{r} a_{s} \\
& =\left(a_{m}\right)_{n-2} a_{r} a_{s} \cdot a_{i}\left(a_{m}\right)_{n-1}=\overline{a_{m}}, \\
& \left(a_{m}\right)_{n-1} a_{j} \cdot\left(a_{m}\right)_{n-2} a_{r} a_{s} \\
& =a\left(a_{m}\right)_{n-2} a_{r} a_{s} \cdot\left(a_{m}\right)_{n-1} a_{j}=\overline{a_{m}}, \\
& \overline{a_{m}} \cdot a_{p} a_{q}\left(a_{m}\right)_{n-2}=a_{p} a_{q}\left(a_{m}\right)_{n-2} \cdot \overline{a_{m}}=\overline{a_{m}}, \\
& \overline{a_{m}} \cdot a_{p}\left(a_{m}\right)_{n-2} a_{s}=a_{p}\left(a_{m}\right)_{n-2} a_{s} \cdot \overline{a_{m}}=\overline{a_{m}}, \\
& \overline{a_{m}} \cdot\left(a_{m}\right)_{n-2} a_{r} a_{s}=\left(a_{m}\right)_{n-2} a_{r} a_{s} \cdot \overline{a_{m}}=\overline{a_{m}} .
\end{aligned}
$$


TABLE 1

\begin{tabular}{lll}
\hline $\mathscr{D}_{\mathcal{N}_{m}^{2}}^{2}$ & $\mathscr{D}_{t}^{2}$ \\
\hline$\alpha_{1}=\overline{a_{m}}$ & $\beta_{1}=\overline{a_{t}}$ \\
$\alpha_{2}=a_{i}\left(a_{m}\right)_{n-1}, \quad i=0, \ldots, m-1$ & $\beta_{2}=a_{\ell}\left(a_{t}\right)_{n-1}, \quad \ell=0, \ldots, m-1$ \\
$\alpha_{3}=\left(a_{m}\right)_{n-1} a_{j}, \quad j=m+1, \ldots, k-1$ & $\beta_{3}=\left(a_{t}\right)_{n-1} a_{u}, \quad u=m+1, \ldots, k-1$ \\
$\alpha_{4}=a_{p} a_{q}\left(a_{m}\right)_{n-2}, \quad p, q=0, \ldots, m-1$ & $\beta_{4}=a_{h} a_{g}\left(a_{t}\right)_{n-2}, \quad h, g=0, \ldots, m-1$ \\
$\alpha_{5}=\left(a_{m}\right)_{n-2} a_{r} a_{s}, \quad r, s=m+1, \ldots, k-1$ & $\beta_{5}=\left(a_{t}\right)_{n-2} a_{u} a_{v}, \quad u, v=m+1, \ldots, k-1$ \\
$\alpha_{6}=a_{p}\left(a_{m}\right)_{n-2} a_{s}, \quad p=0, \ldots, m-1, s=m+1, \ldots, k-1$ & $\beta_{6}=a_{\ell}\left(a_{t}\right)_{n-2} a_{u}, \quad \ell=0, \ldots, m-1, u=m+1, \ldots, k-1$ \\
\hline
\end{tabular}

Case 4. Let $a_{0}=1$ and $a_{k-1}<n-2$. Then, $1_{2}\left(a_{m}\right)_{n-2}$ is the only idempotent in $\mathscr{D} \mathcal{N}_{m}^{2}$. Additionally, to the equalities of the previous case, we find

$$
\begin{gathered}
1_{2}\left(a_{m}\right)_{n-2} \cdot a_{p} a_{q}(n-2)_{n-2}=\left(a_{q}\right)_{2}\left(a_{m}\right)_{n-2}, \\
1\left(a_{m}\right)_{n-1} \cdot a_{p} a_{q}\left(a_{m}\right)_{n-2}=a_{q}\left(a_{m}\right)_{n-1} .
\end{gathered}
$$

Case 5. Let $1<a_{0}$ and $a_{k-1}=n-2$. Now the only idempotent endomorphism in $\mathscr{D} \mathcal{N}_{m}^{2}$ is $\left(a_{m}\right)_{n-2}(n-2)_{2}$. We additionally find the following equalities:

$$
\begin{gathered}
\left(a_{m}\right)_{n-2}(n-2)_{2} \cdot\left(a_{m}\right)_{n-2} a_{r} a_{s}=\left(a_{m}\right)_{n-2}\left(a_{r}\right)_{2}, \\
\left(a_{m}\right)_{n-1}(n-2) \cdot\left(a_{m}\right)_{n-2} a_{r} a_{s}=\left(a_{m}\right)_{n-1} a_{r} .
\end{gathered}
$$

Case 6. Let $a_{0}=1$ and $a_{k-1}=n-2$. Now, in $\mathscr{D} \mathcal{N}_{m}^{2}$, there are two idempotents: $1_{2}\left(a_{m}\right)_{n-2}$ and $\left(a_{m}\right)_{n-2}(n-2)_{2}$. Here, the equalities from Cases 4 and 5 are valid and also all the equalities from Case 3, under the respective restrictions for the indices, are fulfilled.

Hence, $\mathscr{D} \mathcal{N}_{m}^{2}$ is a semiring.

From Lemma 11, we find the following.

Theorem 12. The union $I=\bigcup_{m=0}^{n-1} \mathscr{D} \mathcal{N}_{m}^{2}$ of the discrete 2neighborhoods with respect to all vertices of internal simplex $\sigma^{(n)}\left\{a_{0}, \ldots, a_{k-1}\right\}$ is a right ideal of the simplex.

Proof. For the endomorphisms of $\mathscr{D} \mathcal{N}_{m}^{2}$ and $\mathscr{D} \mathcal{N}_{t}^{2}$, where $t>$ $m$, from (11), it follows as shown in Table 1

Let $\beta_{i}, i=2,3,4,5,6$, be elements of $\mathscr{D} \mathcal{N}_{t}^{2}$ (see Table 1 ). We denote by $\widetilde{\beta}_{i}$ an endomorphism, which maps the same elements to $a_{t}$, but other images (one or two) are not in $\operatorname{Im}(\alpha)$. For example, $\widetilde{\beta_{2}}=a_{\ell_{0}}\left(a_{t}\right)_{n-1}$, where $\ell_{0}=0, \ldots, m-1$ and $\ell_{0} \neq \ell$. Evidently, $\widetilde{\beta}_{i} \in \mathscr{D} \mathcal{N}_{t}^{2}$, for $i=2,3,4,5,6$.

Now we calculate the following:

(1) $\alpha_{1}+\beta_{1}=\beta_{1}, \alpha_{1}+\beta_{2}=\beta_{2}$ or $\alpha_{1}+\beta_{2}=\widetilde{\beta_{2}}, \alpha_{1}+\beta_{3}=\beta_{3}$, and $\alpha_{1}+\beta_{4}=\beta_{4}$ or $\alpha_{1}+\beta_{4}=\widetilde{\beta}_{4}, \alpha_{1}+\beta_{5}=\beta_{5}$, and $\alpha_{1}+\beta_{6}=\beta_{6}$ or $\alpha_{1}+\beta_{2}=\widetilde{\beta_{6}}$;

(2) $\alpha_{2}+\beta_{1}=\beta_{1}, \alpha_{2}+\beta_{2}=\beta_{2}$ or $\alpha_{2}+\beta_{2}=\widetilde{\beta_{2}}, \alpha_{2}+\beta_{3}=\beta_{3}$, and $\alpha_{2}+\beta_{4}=\beta_{4}$ or $\alpha_{2}+\beta_{4}=\widetilde{\beta_{4}}, \alpha_{2}+\beta_{5}=\beta_{5}$, and $\alpha_{2}+\beta_{6}=\beta_{6}$ or $\alpha_{2}+\beta_{2}=\widetilde{\beta_{6}}$;
(3) $\alpha_{3}+\beta_{1}=\beta_{1}$ or $\alpha_{3}+\beta_{1}=\widetilde{\beta_{3}}, \alpha_{3}+\beta_{2}=\beta_{2}$ or $\alpha_{3}+\beta_{2}=$ $\widetilde{\beta_{2}}, \alpha_{3}+\beta_{3}=\beta_{3}$ or $\alpha_{3}+\beta_{3}=\widetilde{\beta_{3}}, \alpha_{3}+\beta_{4}=\beta_{4}$ or $\alpha_{3}+\beta_{4}=\widetilde{\beta_{4}}, \alpha_{3}+\beta_{5}=\beta_{5}$ or $\alpha_{3}+\beta_{5}=\widetilde{\beta_{5}}$, and $\alpha_{3}+\beta_{6}=\beta_{6}$ or $\alpha_{3}+\beta_{6}=\widetilde{\beta_{6}} ;$

(4) $\alpha_{4}+\beta_{1}=\beta_{1}, \alpha_{4}+\beta_{2}=\beta_{2}$ or $\alpha_{4}+\beta_{2}=\widetilde{\beta_{2}}, \alpha_{4}+\beta_{3}=\beta_{3}$, and $\alpha_{4}+\beta_{4}=\beta_{4}$ or $\alpha_{4}+\beta_{4}=\widetilde{\beta_{4}}, \alpha_{4}+\beta_{5}=\beta_{5}$ and $\alpha_{4}+\beta_{6}=\beta_{6}$ or $\alpha_{4}+\beta_{6}=\widetilde{\beta_{6}}$;

(5) $\alpha_{5}+\beta_{1}=\beta_{1}$ or $\alpha_{5}+\beta_{1}=\widetilde{\beta_{3}}$ or $\alpha_{5}+\beta_{1}=\widetilde{\beta_{5}}, \alpha_{5}+\beta_{2}=\beta_{2}$ or $\alpha_{5}+\beta_{2}=\widetilde{\beta_{2}}$ or $\alpha_{5}+\beta_{2}=\widetilde{\beta_{5}}$ or $\alpha_{5}+\beta_{2}=\widetilde{\beta_{6}}$, $\alpha_{5}+\beta_{3}=\beta_{3}$ or $\alpha_{5}+\beta_{3}=\widetilde{\beta_{3}}$ or $\alpha_{5}+\beta_{3}=\widetilde{\beta_{5}}, \alpha_{5}+\beta_{4}=\beta_{4}$ or $\alpha_{5}+\beta_{4}=\widetilde{\beta_{4}}$ or $\alpha_{5}+\beta_{4}=\widetilde{\beta_{5}}$ or $\alpha_{5}+\beta_{4}=\widetilde{\beta}_{6}$, $\alpha_{5}+\beta_{5}=\beta_{5}$ or $\alpha_{5}+\beta_{5}=\widetilde{\beta_{5}}$ and $\alpha_{5}+\beta_{6}=\beta_{6}$ or $\alpha_{5}+\beta_{6}=\widetilde{\beta_{5}}$ or $\alpha_{5}+\beta_{6}=\widetilde{\beta_{6}}$;

(6) $\alpha_{6}+\beta_{1}=\beta_{1}$ or $\alpha_{6}+\beta_{1}=\widetilde{\beta_{3}}, \alpha_{6}+\beta_{2}=\beta_{2}$ or $\alpha_{6}+\beta_{2}=$ $\widetilde{\beta_{2}}, \alpha_{6}+\beta_{3}=\beta_{3}$ or $\alpha_{6}+\beta_{3}=\widetilde{\beta_{3}}$ or $\alpha_{6}+\beta_{3}=\widetilde{\beta_{5}}$, $\alpha_{6}+\beta_{4}=\beta_{4}$ or $\alpha_{6}+\beta_{4}=\widetilde{\beta_{4}}$ or $\alpha_{6}+\beta_{4}=\widetilde{\beta_{6}}, \alpha_{6}+\beta_{5}=\beta_{5}$ or $\alpha_{6}+\beta_{5}=\widetilde{\beta_{5}}$ and $\alpha_{6}+\beta_{6}=\beta_{6}$ or $\alpha_{6}+\beta_{6}=\widetilde{\beta_{5}}$ or $\alpha_{6}+\beta_{6}=\widetilde{\beta_{6}}$.

From these calculations and Lemma 11 we conclude that $I$ is closed under the addition.

Let $\alpha \in I$ and $\varphi \in \sigma^{(n)}\left\{a_{0}, \ldots, a_{k-1}\right\}$. Then, $\alpha \in \mathscr{D} \mathcal{N}_{m}^{2}$, for some $m=0, \ldots, k-1$. Considering the cases where $\alpha=\alpha_{i}$, $i=1,2,3$, we consider in the proof of Theorem 9. Now we consider three new cases.

Case 1. Let $\alpha=\alpha_{4}=a_{p} a_{q}\left(a_{m}\right)_{n-2}$, where $p, q=0, \ldots, m-1$. Let $\varphi\left(a_{m}\right)=a_{t}, \varphi\left(a_{p}\right)=a_{p_{0}}$, and $\varphi\left(a_{q}\right)=a_{q_{0}}$. Then, $\alpha \cdot \varphi=$ $a_{p_{0}} a_{q_{0}}\left(a_{t}\right)_{n-2} \in I$. The same is true when $q_{0}=t$ or $p_{0}=q_{0}=t$.

Case 2. Let $\alpha=\alpha_{5}=\left(a_{m}\right)_{n-2} a_{r} a_{s}$, where $r, s=m+1, \ldots, k-1$. Let $\varphi\left(a_{m}\right)=a_{t}, \varphi\left(a_{r}\right)=a_{r_{0}}$, and $\varphi\left(a_{s}\right)=a_{s_{0}}$. Then $\alpha \cdot \varphi=$ $\left(a_{t}\right)_{n-2} a_{r_{0}} a_{s_{0}} \in I$. The same is true when $r_{0}=t$ or $s_{0}=r_{0}=t$.

Case 3. Let $\alpha=\alpha_{6}=a_{p}\left(a_{m}\right)_{n-2} a_{s}$, where $p=0, \ldots, m-1$ and $s=m+1, \ldots, k-1$. Let $\varphi\left(a_{m}\right)=a_{t}, \varphi\left(a_{p}\right)=a_{p_{0}}$, and $\varphi\left(a_{s}\right)=a_{s_{0}}$. Then, $\alpha \cdot \varphi=a_{p_{0}}\left(a_{t}\right)_{n-2} a_{s_{0}} \in I$. The same is true when $p_{0}=t, s_{0}=t$, or $p_{0}=s_{0}=t$.

Hence, in all cases, $\alpha \cdot \varphi \in I$.

From Theorem 9 and Theorem 12, we obtain the following. 
Corollary 13. (a) If $\sigma^{(n)}\left\{a_{0}, \ldots, a_{k-1}\right\}$ is an internal simplex, then the union $J=\bigcup_{m=0}^{n-1} \mathscr{D} \mathcal{N}_{m}^{1}$ of the discrete 1-neighborhoods with respect to all vertices is an ideal of the simplex.

(b) Let $\sigma^{(n)}\left\{x_{0}, \ldots, x_{s-1}\right\}$ be an internal simplex and $\sigma^{(n)}\left\{a_{0}, \ldots, a_{k-1}\right\}$ is internal of the simplex $\sigma^{(n)}\left\{x_{0}, \ldots, x_{s-1}\right\}$. Then, the union $I=\bigcup_{m=0}^{n-1} \mathscr{D} \mathcal{N}_{m}^{2}$ of the discrete 2neighborhoods is an ideal of the simplex $\sigma^{(n)}\left\{a_{0}, \ldots, a_{k-1}\right\}$.

Proof. (a) If $\sigma^{(n)}\left\{a_{0}, \ldots, a_{k-1}\right\}$ is an internal simplex and $\varphi \epsilon$ $\sigma^{(n)}\left\{a_{0}, \ldots, a_{k-1}\right\}$, then $\varphi(0)>0$ and $\varphi(n-1)<n-1$. We calculate $\varphi \cdot \overline{a_{m}}=\varphi \cdot a_{i}\left(a_{m}\right)_{n-1}=\varphi \cdot\left(a_{m}\right)_{n-1} a_{j}=\overline{a_{m}}$.

From Theorem 9, it follows that $J$ is an ideal of the simplex $\sigma^{(n)}\left\{a_{0}, \ldots, a_{k-1}\right\}$.

(b) If $\sigma^{(n)}\left\{a_{0}, \ldots, a_{k-1}\right\}$ satisfies the condition of Theorem 12 and $\varphi \in \sigma^{(n)}\left\{a_{0}, \ldots, a_{k-1}\right\}$, then $\varphi(0)>1$ and $\varphi(n-1)<n-2$. Thus (see the notations in the proof of Theorem 12) we obtain

$$
\varphi \cdot \alpha_{1}=\varphi \cdot \alpha_{2}=\varphi \cdot \alpha_{3}=\varphi \cdot \alpha_{4}=\varphi \cdot \alpha_{5}=\varphi \cdot \alpha_{6}=\overline{a_{m}} .
$$

From Theorem 12, it follows that $I$ is an ideal of the simplex $\sigma^{(n)}\left\{a_{0}, \ldots, a_{k-1}\right\}$.

Proposition 14. Let $\sigma_{k}^{(n)}(A)=\sigma^{(n)}\left\{a_{0}, a_{1}, \ldots, a_{k-1}\right\}$ be a simplex.

(a) For the least vertex $\overline{a_{0}}$, it follows that $\mathscr{D} \mathscr{N}_{0}^{n-a_{0}-1}=$ $\sigma_{k}^{(n)}(A) \cap \mathscr{E}_{\mathscr{C}_{n}}^{\left(a_{0}\right)}$.

(b) For the biggest vertex $\overline{a_{k-1}}$, it follows that $\mathscr{D} \mathscr{N}_{k-1}^{a_{k-1}}=$ $\sigma_{k}^{(n)}(A) \cap \mathscr{E}_{\mathscr{C}_{n}}^{\left(a_{k-1}\right)}$.

Proof. (a) Since $\overline{a_{0}}$ is the least vertex of the simplex, it follows that layer $\mathscr{L}_{a_{0}}^{a_{0}+1}\left(\sigma^{(n)}\left\{a_{0}, a_{1}, \ldots, a_{k-1}\right\}\right)$ consists of endomorphisms $\alpha=\left(a_{0}\right)_{a_{0}+1}\left(a_{1}\right)_{p_{1}} \cdots\left(a_{k-1}\right)_{p_{k-1}}$, where $a_{0}+1+p_{1}+$ $\cdots+p_{k-1}=n$; that is, $\alpha(0)=a_{0}, \ldots, \alpha\left(a_{0}\right)=a_{0}$. All the layers $\mathscr{L}_{a_{0}}^{\ell}\left(\sigma^{(n)}\left\{a_{0}, a_{1}, \ldots, a_{k-1}\right\}\right)$, where $\ell \geq a_{0}+1$, consist of endomorphisms having $a_{0}$ as a fixed point. So, $\mathscr{D} \mathcal{N}_{0}^{n-a_{0}-1} \subseteq$ $\sigma_{k}^{(n)}(A) \cap \mathscr{E}_{\mathscr{C}_{n}}^{\left(a_{0}\right)}$.

Conversely, let $\alpha \in \sigma_{k}^{(n)}(A) \cap \mathscr{E}_{\mathscr{C}_{n}}^{\left(a_{0}\right)}$. Then, $\alpha\left(a_{0}\right)=a_{0}$. Since $\overline{a_{0}}$ is the least vertex of the simplex, we have $\alpha(0)=\cdots=$ $\alpha\left(a_{0}-1\right)=a_{0}$; that is, $\alpha \in \mathscr{L}_{a_{0}}^{\ell}\left(\sigma^{(n)}\left\{a_{0}, a_{1}, \ldots, a_{k-1}\right\}\right)$, where $\ell \geq a_{0}+1$. Hence, $\mathscr{D} \mathcal{N}_{0}^{n-a_{0}-1}=\sigma_{k}^{(n)}(A) \cap \mathscr{E}_{\mathscr{C}_{n}}^{\left(a_{0}\right)}$.

(b) Since $\overline{a_{k-1}}$ is the biggest vertex of the simplex, it follows that layer $\mathscr{L}_{a_{k-1}-a_{k-1}}\left(\sigma^{(n)}\left\{a_{0}, a_{1}, \ldots, a_{k-1}\right\}\right)$ consists of endomorphisms $\alpha=\left(a_{0}\right)_{p_{0}} \ldots\left(a_{k-2}\right)_{p_{k-2}}\left(a_{k-1}\right)_{n-a_{k-1}}$, where $p_{0}+\cdots+p_{k-2}+n-a_{k-1}=n$. So, $p_{0}+\cdots+p_{k-2}=a_{k-1}$ implies that the images of $0, \ldots, a_{k-1}-1$ are not equal to $a_{k-1}$, but $\alpha\left(a_{k-1}\right)=a_{k-1}$. For all the endomorphisms of layers $\mathscr{L}_{a_{k-1}}^{\ell}\left(\sigma^{(n)}\left\{a_{0}, a_{1}, \ldots, a_{k-1}\right\}\right)$, where $\ell \geq n-a_{k-1}$, we have $p_{0}+\cdots+p_{k-2}=a_{k-1}$. Hence, the elements of these layers have $a_{k-1}$ as a fixed point and $\mathscr{D} \mathscr{N}_{0}^{a_{k-1}} \subseteq \sigma_{k}^{(n)}(A) \cap \mathscr{E}_{\mathscr{C}_{n}}^{\left(a_{k-1}\right)}$.
Conversely, let $\alpha \in \sigma_{k}^{(n)}(A) \cap \mathscr{E}_{\mathscr{C}_{n}}^{\left(a_{k-1}\right)}$. Then, $\alpha\left(a_{k-1}\right)=$ $a_{k-1}$. Since $\overline{a_{k-1}}$ is the biggest vertex of the simplex, we have $\alpha\left(a_{k-1}+1\right)=\cdots=\alpha(n-1)=a_{k-1}$. Thus, it follows that $\alpha \in \mathscr{L}_{a_{k-1}}^{\ell}\left(\sigma^{(n)}\left\{a_{0}, a_{1}, \ldots, a_{k-1}\right\}\right)$, where $\ell \geq n-a_{k-1}$.

Hence, $\mathscr{D} \mathcal{N}_{k-1}^{a_{k-1}}=\sigma_{k}^{(n)}(A) \cap \mathscr{E}_{\mathscr{C}_{k-1}}^{\left(a_{k-1}\right)}$.

\section{A Partition of a Simplex}

Let $\sigma^{(n)}\left\{a_{0}, a_{1}, \ldots, a_{k-1}\right\}$ be a simplex. Then, $\alpha \in \sigma^{(n)}\left\{a_{0}\right.$, $\left.a_{1}, \ldots, a_{k-1}\right\}$ is called endomorphism of type $\left\langle\left\langle m_{0}\right.\right.$, $\left.\left.\ldots, m_{k-1}\right\rangle\right\rangle$, where $m_{i} \in\{0, \ldots, k-1\}, m_{i} \leq m_{j}$ for $i<j, i, j=0, \ldots, k-1$, if $\alpha\left(a_{i}\right)=a_{m}$.

Obviously, the relation $\alpha \sim \beta$, if and only if $\alpha$ and $\beta$ are of the same type, is an equivalence relation. Any equivalence class is closed under the addition. But there are equivalence classes which are not closed under the multiplication. For example, consider $\alpha=\left(a_{0}\right)_{a_{1}+1}\left(a_{1}\right)_{a_{2}-a_{1}}\left(a_{2}\right)_{n-a_{2}-1}$, where $n>$ $a_{3}>a_{2}+1$. Since $\alpha\left(a_{0}\right)=a_{0}, \alpha\left(a_{1}\right)=a_{0}, \alpha\left(a_{2}\right)=$ $a_{1}$, and $\alpha\left(a_{3}\right)=a_{2}, \ldots, \alpha\left(a_{k-1}\right)=a_{2}$, the type of $\alpha$ is $\langle\langle 0,0,1,2, \ldots, 2\rangle\rangle$. But $\alpha^{2}=\left(a_{0}\right)_{a_{2}+1}\left(a_{1}\right)_{n-a_{2}-1}$ is of type $\langle\langle 0,0,0,1, \ldots, 1\rangle\rangle$.

Sometimes it is possible to describe the semiring structure of union of many equivalence classes, that is, blocks of our partition. For example, the union of endomorphisms from all the blocks of type $\langle\langle 0, *, \ldots, *\rangle\rangle$ is the set of $\alpha \in \sigma^{(n)}\left\{a_{0}, a_{1}, \ldots, a_{k-1}\right\}$ such that $\alpha\left(a_{0}\right)=a_{0}$ and, from Proposition 14 , it is the semiring $\mathscr{D} \mathscr{N}_{0}^{n-a_{0}-1}$.

The type of any endomorphism is itself an endomorphism of a simplex $\widehat{\mathscr{C}}_{\mathscr{C}_{k}}=\sigma^{(k)}\{0,1, \ldots, k-1\}$. The simplex $\widehat{\mathscr{C}}_{\mathscr{C}_{k}}$ is called a coordinate simplex of $\sigma^{(n)}\left\{a_{0}, a_{1}, \ldots, a_{k-1}\right\}$. From this point of view, the set of endomorphisms from all the blocks of type $\langle\langle 0, *, \ldots, *\rangle\rangle$ really corresponds to the set of all endomorphisms $20, m_{1}, \ldots, m_{k-1} \imath \in \overline{\mathscr{E}}_{\mathscr{C}_{k}}$, which is the semiring $\mathscr{E}_{\mathscr{C}_{k}}^{(0)}$. More generally, we can consider the semiring $\mathscr{C}_{\mathscr{C}_{k}}^{(j)}$, where $j \in \mathscr{C}_{k}$, consisting of all $\varphi \in \widehat{\mathscr{C}}_{\mathscr{C}_{k}}$ such that $\varphi(j)=j$. Then, $\alpha\left(a_{j}\right)=a_{m_{j}}=a_{\varphi(j)}=a_{j}$. So the union of endomorphisms from all the blocks of type $\langle\langle *, \ldots, *, j, *, \ldots, *\rangle\rangle$ is semiring $\sigma^{(n)}\left\{a_{0}, a_{1}, \ldots, a_{k-1}\right\} \cap$ $\mathscr{E}_{\mathscr{C}_{n}}^{\left(a_{j}\right)}$. Now, more generally again, we can consider the semiring $\bigcap_{s=1}^{m} \mathscr{E}_{\mathscr{C}_{k}}^{\left(j_{s}\right)}$ consisting of all endomorphisms of $\widehat{\mathscr{E}}_{\mathscr{C}_{k}}$ having $j_{1}, \ldots, j_{m}$ as fixed points. Then, similarly, $\alpha\left(a_{j_{s}}\right)=a_{j_{s}}$, for all $s=1, \ldots, m$. So the union of endomorphisms from all the blocks of type $\left\langle\left\langle *, \ldots, *, j_{1}, *, \ldots, *, j_{m}, *, \ldots, *\right\rangle\right\rangle$ is semiring $\sigma^{(n)}\left\{a_{0}, a_{1}, \ldots, a_{k-1}\right\} \cap\left(\bigcap_{s=1}^{m} \mathscr{E}_{\mathscr{C}_{n}}^{\left(a_{j_{s}}\right)}\right)$. The next results from this section are announced in [8].

Theorem 15. Let $\sigma^{(n)}\left\{a_{0}, \ldots, a_{k-1}\right\}$ be a simplex. Let $R$ be a subsemiring of the coordinate simplex $\widehat{\mathscr{E}}_{\mathscr{C}_{k}}$ of $\sigma^{(n)}\left\{a_{0}, \ldots, a_{k-1}\right\}$. The set $\widetilde{R}$ of endomorphisms of $\sigma^{(n)}\left\{a_{0}, \ldots, a_{k-1}\right\}$, of type $\left\langle\left\langle m_{0}, \ldots, m_{k-1}\right\rangle\right\rangle$, where the endomorphism $\left.2 m_{0}, \ldots, m_{k-1}\right\rangle \in$ $R$, is a semiring. Moreover, when $R$ is a (right, left) ideal of semiring $\widehat{\mathscr{C}}_{\mathscr{C}_{k}}$, it follows that $\widetilde{R}$ is a (right, left) ideal of simplex $\sigma^{(n)}\left\{a_{0}, \ldots, a_{k-1}\right\}$. 
Proof. Let $\alpha, \beta \in \sigma^{(n)}\left\{a_{0}, \ldots, a_{k-1}\right\}$. Let $\alpha$ be of type $\left\langle\left\langle p_{0}, \ldots, p_{k-1}\right\rangle\right\rangle$, where the endomorphism $\varphi=\imath p_{0}, \ldots$, $p_{k-1}{ } \in R$ and similarly $\beta$ are of type $\left\langle\left\langle q_{0}, \ldots, q_{k-1}\right\rangle\right\rangle$, where the endomorphism $\psi=2 q_{0}, \ldots, q_{k-1}{ }^{2} \in R$. Then, we find $(\alpha+\beta)\left(a_{i}\right)=\alpha\left(a_{i}\right)+\beta\left(a_{i}\right)=a_{p_{i}}+a_{q_{i}}=a_{m_{i}}$, where $m_{i}=\max \left\{p_{i}, q_{i}\right\}$. But the endomorphism $2 m_{0}, \ldots, m_{k-1} 2$ is the sum $\varphi+\psi$. So we prove that endomorphism $\alpha+\beta$ is of type $\varphi+\psi \in R$; that is, $\alpha+\beta \in \widetilde{R}$.

Now, let us assume that $\varphi$ and $\psi$ are arbitrary endomorphisms of $\widehat{\mathscr{E}}_{\mathscr{C}_{k}}$. Then, we find

$$
\begin{aligned}
(\alpha \cdot \beta)\left(a_{i}\right) & =\beta\left(\alpha\left(a_{i}\right)\right)=\beta\left(a_{m_{i}}\right) \\
& =\beta\left(a_{\varphi(i)}\right)=a_{\psi(\varphi(i))}=a_{(\varphi \cdot \psi)(i)} .
\end{aligned}
$$

So, if $R$ is a right ideal of $\widehat{\mathscr{E}}_{\mathscr{C}_{k}}$ and $\varphi \in R, R$ is a left ideal of $\widehat{\mathscr{E}}_{\mathscr{C}_{k}}$ and $\psi \in R$, or $R$ is an ideal of $\widehat{\mathscr{E}}_{\mathscr{C}_{k}}$ and one of $\varphi$ and $\psi$ is from $R$, it follows that $\varphi \cdot \psi \in R$. Hence, in each of the three cases $\alpha \cdot \beta \in \widetilde{R}$ and this completes the proof.

Now, let endomorphism $\alpha$ be of type $\left\langle\left\langle\iota_{0}, \ldots, \iota_{k-1}\right\rangle\right\rangle$ and the endomorphism $\iota=\imath \iota_{0}, \ldots, \iota_{k-1}$ ? from the coordinate simplex $\widehat{\mathscr{E}}_{\mathscr{C}_{k}}$ be an idempotent, different from constant endomorphisms $\bar{j}$, where $j=0, \ldots, k-1$, and the identity i. Then we say that $\alpha$ is of an idempotent type.

Corollary 16. The set of endomorphisms $\alpha \in \sigma^{(n)}$ $\left\{a_{0}, \ldots, a_{k-1}\right\}$ of a fixed idempotent type $\left\langle\left\langle\iota_{0}, \ldots, \iota_{k-1}\right\rangle\right\rangle$ is a semiring.

Proof. Obviously, since the set $R=\{l\}$, where $\iota$ is an idempotent, is a semiring, the semirings of an idempotent type are denoted by $\widetilde{R}=\mathscr{I}(\iota)$, where $\iota=\imath \iota_{0}, \ldots, \iota_{k-1}$ 2 is the corresponding idempotent.

Let $\ell \in \mathscr{C}_{k}$. For any $\bar{\ell}=\imath \ell, \ldots, \ell \imath \in \widehat{\mathscr{C}}_{\mathscr{C}_{k}}$, we consider (see the first section) the set

$$
\begin{aligned}
\mathcal{N}_{k}^{[\ell]}=\left\{\varphi \mid \varphi \in \widehat{\mathscr{E}}_{\mathscr{C}_{k}},\right. \\
\left.\qquad \varphi^{m_{\ell}}=\bar{\ell} \text { for some natural number } m_{\ell}\right\} .
\end{aligned}
$$

From Theorem 1, it follows that $\mathcal{N}_{k}^{[\ell]}$, for $k \geq 2$ and $\ell \epsilon$ $\mathscr{C}_{k}$, is a subsemiring of $\widehat{\mathscr{C}}_{\mathscr{C}_{k}}$.

Now, let the endomorphism $\alpha \in \sigma^{(n)}\left\{a_{0}, \ldots, a_{k-1}\right\}$ be of any type $\left\langle\left\langle m_{0}, \ldots, m_{k-1}\right\rangle\right\rangle$, where the endomorphism $2 m_{0}, \ldots, m_{k-1} \imath \in \mathcal{N}_{k}^{[\ell]}$, for some fixed $\ell \in \mathscr{C}_{k}$. Then, we say that $\alpha$ is of an $\ell$-nilpotent type.

Corollary 17. The set of endomorphisms $\alpha \in \sigma^{(n)}$ $\left\{a_{0}, \ldots, a_{k-1}\right\}$ of $\ell$-nilpotent type, for some fixed $\ell \in \mathscr{C}_{k}$, is a semiring.

Proof. Immediately from the last theorem and Theorem 1 , the semirings of $\ell$-nilpotent type are denoted by $\widetilde{R}=$ $\mathcal{N}^{\left[a_{\ell}\right]}\left(\sigma^{(n)}\left\{a_{0}, \ldots, a_{k-1}\right\}\right)$.
Now, let the endomorphism $\alpha \in \sigma^{(n)}\left\{a_{0}, \ldots, a_{k-1}\right\}$ be of any type $\left\langle\left\langle m_{0}, \ldots, m_{k-1}\right\rangle\right\rangle$, where the endomorphism $\varphi=$ $2 m_{0}, \ldots, m_{k-1} 2$ from the coordinate simplex $\widehat{\mathscr{E}}_{\mathscr{C}_{k}}$ is neither an idempotent nor an $\ell$-nilpotent for some $\ell \in \mathscr{C}_{k}$. Then, according to [5], $\varphi$ is a root of some idempotent $\iota=$ $\imath \iota_{0}, \ldots, \iota_{k-1} 2$. Since the roots of identity of semiring $\widehat{\mathscr{E}}_{\mathscr{C}_{k}}$ do not exist (see [2]), it follows that $\iota$ is an idempotent, different from $\bar{\ell}, \ell \in \mathscr{C}_{k}$, and identity. In this case, the endomorphism $\alpha$ is called a type related to idempotent type $\left\langle\left\langle\iota_{0}, \ldots, \iota_{k-1}\right\rangle\right\rangle$. To clarify the above definition, we give an example of endomorphisms of type related to some idempotent type.

Example 18. Let us consider the simplex $\sigma^{(10)}\{0,2,3,5,8\}$. The coordinate simplex consisting of all the types of endomorphisms of $\sigma^{(10)}\{0,2,3,5,8\}$ is the simplex $\sigma^{(5)}\{0,1,2,3,4\}$. In this coordinate simplex, we chose the idempotent $\iota=$

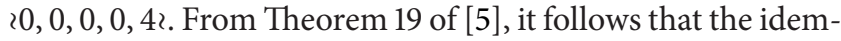
potent $\iota$ and its roots form a semiring of order $C_{3}=5$. Let us note that Catalan sequence is the sequence $C_{0}, C_{1}, C_{2}, C_{3}, \ldots$, where $C_{n}=1 /(n+1)\left(\begin{array}{c}2 n \\ n\end{array}\right)$. So, the elements of the semiring, generated by $\iota$ contains 5 endomorphisms: $\varphi_{1}=$ $\imath 0,0,0,1,4 \imath, \varphi_{2}=\imath 0,0,0,2,4 \imath, \varphi_{3}=\imath 2,0,1,1,4 \imath, \varphi_{4}=$ $20,0,1,2,42$, and $\iota$. Now, we choose endomorphisms from the simplex $\sigma^{(10)}\{0,2,3,5,8\}: \alpha=0_{4} 2_{2} 8_{4}$ and $\beta=0_{3} 2_{2} 3_{3} 8_{5}$. The endomorphism $\alpha$ is of type $\langle\langle 0,0,0,1,4\rangle\rangle$, related to idempotent type $\langle\langle 0,0,0,0,4\rangle\rangle$, and endomorphism $\beta$ is of type $\langle\langle 0,0,1,2,4\rangle\rangle$, related to the same idempotent type. We compute $\alpha^{2}=0_{6} 8_{4}$ which is an idempotent and also $\beta^{2}=0_{5} 2_{3} 8_{2}$ which is not an idempotent, but $\beta^{3}=0_{8} 8_{2}$ is an idempotent. Thus, we show that $\alpha$ and $\beta$ are roots of different idempotents, but they are of types related to the same idempotent type. We can also show that $\alpha \cdot \beta=0_{6} 8_{4}$ is an idempotent and $\beta \cdot \alpha=0_{8} 8_{2}$ also is an idempotent.

Corollary 19. The set of endomorphisms $\alpha \in \epsilon$ $\sigma^{(n)}\left\{a_{0}, \ldots, a_{k-1}\right\}$ of a type, related to some fixed idempotent type, is a semiring.

Proof. Immediately from the last theorem and Theorem 19 of [5], the semirings from the last corollary are called idempotent closures of type $\iota$, where $\iota=\imath \iota_{0}, \ldots, \iota_{k-1}$ is the corresponding idempotent and is denoted by $\widetilde{R}=\mathscr{I} \mathscr{C}(\iota)$.

From the last theorem, we also find the following.

Corollary 20. The set of endomorphisms of $\sigma^{(n)}\left\{a_{0}, \ldots, a_{k-1}\right\}$, of type $\left\langle\left\langle m_{0}, \ldots, m_{k-1}\right\rangle\right\rangle$, where the endomorphism $2 m_{0}, \ldots, m_{k-1}{ }^{2}$ belongs to some face of the coordinate simplex $\widehat{\mathscr{E}}_{\mathscr{C}_{k}}$, is a left ideal.

Now we describe the left ideals from the last corollary when $k=4$.

Example 21. For a simplex $\mathscr{T} \mathscr{E} \mathscr{T} \mathscr{R}^{(n)}\left\{a_{0}, a_{1}, a_{2}, a_{3}\right\}$, the coordinate simplex is $\mathscr{T} \mathscr{E} \mathscr{T} \mathscr{R}^{(4)}\{0,1,2,3\}$ and its faces are as follows:

$$
\text { triangles: } I_{0}=\Delta^{(4)}\{1,2,3\}, I_{1}=\triangle^{(4)}\{0,2,3\}, I_{2}=
$$
$\triangle^{(4)}\{0,1,3\}$, and $I_{3}=\Delta^{(4)}\{0,1,2\}$; 
strings: $I_{0} \cap I_{1}=\mathcal{S} \mathscr{T} \mathscr{R}^{(4)}\{2,3\}, I_{0} \cap I_{2}=$ $\mathcal{S} \mathscr{T} \mathscr{R}^{(4)}\{1,3\}, I_{0} \cap I_{3}=\mathcal{S} \mathscr{T} \mathscr{R}^{(4)}\{1,2\}, I_{1} \cap I_{2}=$ $\mathcal{S} \mathscr{T} \mathscr{R}^{(4)}\{0,3\}, I_{1} \cap I_{3}=\mathcal{S} \mathscr{T} \mathscr{R}^{(4)}\{0,2\}$, and $I_{2} \cap I_{3}=$ $\mathcal{S} \mathscr{T} \mathscr{R}^{(4)}\{0,1\}$;

vertices: $I_{0} \cap I_{1} \cap I_{2}=\{\overline{3}\}, I_{0} \cap I_{1} \cap I_{3}=\{\overline{2}\}, I_{0} \cap I_{2} \cap I_{3}=$ $\{\overline{1}\}$, and $I_{1} \cap I_{2} \cap I_{3}=\{\overline{0}\}$.

Then, the left ideal $\widetilde{I_{0}}$ of simplex $\mathscr{T} \mathscr{C} \mathscr{T} \mathscr{R}^{(n)}\left\{a_{0}, a_{1}, a_{2}, a_{3}\right\}$ consists of endomorphisms $\alpha$, such that $a_{0}$ is not a fixed point of $\alpha$. Now, the triangle $\Delta^{(n)}\left\{a_{1}, a_{2}, a_{3}\right\}$, which is a left ideal of semiring $\mathscr{T} \mathscr{C} \mathscr{T} \mathscr{R}^{(n)}\left\{a_{0}, a_{1}, a_{2}, a_{3}\right\}$, is contained in $\widetilde{I_{0}}$. Moreover,

$$
\begin{aligned}
\widetilde{I_{0} \backslash \triangle^{(n)}}\left\{a_{1}, a_{2}, a_{3}\right\} \\
=\bigcup_{s=1}^{a_{0}} \mathscr{L}_{a_{0}}^{s}\left(\mathscr{T} \mathscr{E} \mathscr{T} \mathscr{R}^{(n)}\left\{a_{0}, a_{1}, a_{2}, a_{3}\right\}\right) \\
=\left\{\alpha=\left(a_{0}\right)_{\ell}\left(a_{1}\right)_{p_{1}}\left(a_{2}\right)_{p_{2}}\left(a_{3}\right)_{p_{3}},\right. \\
\left.\quad \text { where } 0<\ell \leq a_{0}, \ell+p_{1}+p_{2}+p_{3}=n\right\} .
\end{aligned}
$$

Similarly, the left ideals $\widetilde{I_{1}}, \widetilde{I_{2}}$ И $\widetilde{I_{3}}$ consist of endomorphisms $\alpha$, such that $a_{1}, a_{2}$, and $a_{3}$ are not fixed points of $\alpha$, respectively.

The left ideals $\widetilde{I_{p}} \cap \widetilde{I_{q}}$, where $p, q \in\{0,1,2,3\}, p \neq q$, consists of endomorphisms $\alpha$, such that $a_{p}$ and $a_{q}$ are not fixed points of $\alpha$. Let $\left\{a_{r}, a_{s}\right\}=\left\{a_{0}, a_{1}, a_{2}, a_{3}\right\} \backslash\left\{a_{p}, a_{q}\right\} И a_{r}<$ $a_{s}$. Then, $\mathcal{S} \mathscr{T} \mathscr{R}^{(4)}\left\{a_{r}, a_{s}\right\} \subset \widetilde{I_{p}} \cap \widetilde{I}_{q}$. Observe that in the interior of one of the triangles with vertices $\overline{a_{p}}, \overline{a_{r}}$, and $\overline{a_{s}}$ and $\overline{a_{q}}, \overline{a_{r}}$, and $\overline{a_{s}}$, respectively, there are endomorphisms $\alpha$, such that $a_{p}$ and $a_{q}$ are not fixed points of $\alpha$.

The left ideal $\widetilde{I}$, where $I=I_{p} \cap I_{q}$ is actually the ideal $\widetilde{I_{p}} \cap \widetilde{I}_{q}$.

The left ideal $\widetilde{I_{0}} \cap \widetilde{I_{1}} \cap \widetilde{I_{2}}$ consists of endomorphisms $\alpha$ such that $a_{0}, a_{1}$, and $a_{2}$ are not fixed points of $\alpha$. Hence, all elements of this left ideal have $a_{3}$ as a fixed point. Similarly, we determine the left ideals $\widetilde{I_{0}} \cap \widetilde{I_{1}} \cap \widetilde{I_{3}}, \widetilde{I_{0}} \cap \widetilde{I_{2}} \cap \widetilde{I_{3}}$, and $\widetilde{I_{1}} \cap \widetilde{I}_{2} \cap \widetilde{I_{3}}$.

From the last theorem, also we have two consequences.

Corollary 22. The set of endomorphisms of the simplex $\sigma^{(n)}\left\{a_{0}, \ldots, a_{k-1}\right\}$, having a type $\left\langle\left\langle a_{i}, \ldots, a_{i}\right\rangle\right\rangle$, where $i=$ $0, \ldots, k-1$, is an ideal.

Corollary 23. The set of endomorphisms of the simplex $\sigma^{(n)}\left\{a_{0}, \ldots, a_{k-1}\right\}$, having a type $\left\langle\left\langle m_{0}, \ldots, m_{k-1}\right\rangle\right\rangle$, where the endomorphism $2 m_{0}, \ldots, m_{k-1}$ ? belongs to the union $J=$ $\bigcup_{m=0}^{k-1} \mathscr{D} \mathscr{N}_{m}^{1}$ of the discrete 1-neighborhoods of all vertices of coordinate simplex $\widehat{\mathscr{E}}_{\mathscr{C}_{k}}$, is a right ideal.

At last, we consider the endomorphisms $\alpha \epsilon$ $\sigma^{(n)}\left\{a_{0}, \ldots, a_{k-1}\right\}$ of type $\langle\langle 0,1, \ldots, k-1\rangle\rangle$. Now the corresponding endomorphism from the coordinate simplex is identity i. In order to find the set of endomorphisms $\widetilde{R}$ of this type, we need the following definition. Idempotent $\alpha \in \sigma^{(n)}\left\{a_{0}, \ldots, a_{k-1}\right\}$ is called a boundary idempotent of the simplex, if $\alpha \in \mathscr{B} \mathscr{D}\left(\sigma^{(n)}\left\{a_{0}, \ldots, a_{k-1}\right\}\right)$, similarly, an interior idempotent of the simplex, if $\alpha \in \mathscr{I} \mathscr{N} \mathscr{T}\left(\sigma^{(n)}\left\{a_{0}, \ldots, a_{k-1}\right\}\right)$. Note that, in the coordinate simplex, the identity $\mathbf{i}$ is the unique interior idempotent.

Theorem 24. The set of endomorphisms of $\sigma^{(n)}\left\{a_{0}, \ldots, a_{k-1}\right\}$, which are right identities, is a semiring of order $\prod_{i=0}^{k-1}\left(a_{i+1}-a_{i}\right)$.

Proof. Let us denote by $\mathscr{R} \mathscr{I}\left(\sigma^{(n)}\left\{a_{0}, \ldots, a_{k-1}\right\}\right)$ semiring $\sigma^{(n)}\left\{a_{0}, \ldots, a_{k-1}\right\} \cap\left(\bigcap_{i=0}^{k-1} \mathscr{C}_{\mathscr{C}_{n}}^{\left(a_{i}\right)}\right)$. Then, for any element $x \in \mathscr{C}_{n}$ and arbitrary endomorphism $\alpha \in \mathscr{R} \mathscr{I}\left(\sigma^{(n)}\left\{a_{0}, \ldots, a_{k-1}\right\}\right)$, it follows that $\alpha(x)=a_{i}$, for some $i=0, \ldots, k-1$. Then $\alpha^{2}(x)=\alpha\left(a_{i}\right)=a_{i}=\alpha(x)$; that is, $\alpha$ is an idempotent. Since $\alpha=\left(a_{0}\right)_{\ell_{0}} \ldots\left(a_{k-1}\right)_{\ell_{k-1}}$, where $\ell_{i}>0$, for any $i=0, \ldots, k-1$ (if we suppose $\ell_{i}=0$, then $\alpha \notin \mathscr{E}_{\mathscr{C}_{n}}^{\left(a_{i}\right)}$ ), it follows that $\alpha$ is an interior idempotent.

Conversely, let $\alpha$ be an interior idempotent. Then, we can express $\alpha=\left(a_{0}\right)_{\ell_{0}} \cdots\left(a_{k-1}\right)_{\ell_{k-1}}$, where $\ell_{i}>0$, for any $i=0, \ldots, k-1$ (if we suppose $\ell_{i}=0$, then $\alpha \notin$ $\left.\mathscr{I} \mathscr{N} \mathscr{T}\left(\sigma^{(n)}\left\{a_{0}, \ldots, a_{k-1}\right\}\right)\right)$. So, we prove that the semiring $\mathscr{R} \mathcal{F}\left(\sigma^{(n)}\left\{a_{0}, \ldots, a_{k-1}\right\}\right)$ consists of all interior idempotents of the simplex.

Let $\alpha \in \mathscr{R} \mathscr{I}\left(\sigma^{(n)}\left\{a_{0}, \ldots, a_{k-1}\right\}\right)$ and $\beta$ be an arbitrary element of the simplex. For $x \in \mathscr{C}_{n}$, it follows that $\beta(x)=a_{i}$, where $i=0, \ldots, k-1$. Then $(\beta \cdot \alpha)(x)=\alpha(\beta(x))=\alpha\left(a_{i}\right)=$ $a_{i}=\beta(x)$. Hence, $\alpha$ is a right identity of the simplex.

Conversely, let $\alpha$ be a right identity of the simplex. Evidently, $\alpha$ is an idempotent. Assume that, for some $i=$ $0, \ldots, k-1$, we have $a_{i} \notin \operatorname{Im}(\alpha)$. Since for some $\beta \in$ $\sigma^{(n)}\left\{a_{0}, \ldots, a_{k-1}\right\}$ and $x \in \mathscr{C}_{n}$, it follows that $\beta(x)=a_{i}$, where $i=0, \ldots, k-1$, we find $(\beta \cdot \alpha)(x)=\alpha(\beta(x))=\alpha\left(a_{i}\right) \neq a_{i}=$ $\beta(x)$; that is, $\beta \cdot \alpha \neq \beta$, which contradicts that $\alpha$ is a right identity. So, $\operatorname{Im}(\alpha)=\left\{a_{0}, \ldots, a_{k-1}\right\}$; that is, $\alpha$ is an interior idempotent or $\alpha \in \mathscr{R} \mathscr{I}\left(\sigma^{(n)}\left\{a_{0}, \ldots, a_{k-1}\right\}\right)$.

Hence, we prove that $\mathscr{R} \mathscr{I}\left(\sigma^{(n)}\left\{a_{0}, \ldots, a_{k-1}\right\}\right)$ is a semiring of right identities of simplex $\sigma^{(n)}\left\{a_{0}, \ldots, a_{k-1}\right\}$. Since elements of $\mathscr{R} \mathscr{I}\left(\sigma^{(n)}\left\{a_{0}, a_{1}, \ldots, a_{k-1}\right\}\right)$ are all the idempotents with fixed points $a_{0}, \ldots, a_{k-1}$, from Theorem 2 (Section 1), it follows that $\left|\mathscr{R} \mathcal{S}\left(\sigma^{(n)}\left\{a_{0}, \ldots, a_{k-1}\right\}\right)\right|=\prod_{i=0}^{k-1}\left(a_{i+1}-a_{i}\right)$.

So, we can construct a partition of the simplex $\sigma^{(n)}\left\{a_{0}, \ldots, a_{k-1}\right\}$ such that all blocks of this partition are as follows:

(1) semirings $\widetilde{R}=\mathcal{N}^{\left[a_{\ell}\right]}\left(\sigma^{(n)}\left\{a_{0}, \ldots, a_{k-1}\right\}\right)$, where $\ell=$ $0, \ldots, k-1$

(2) semirings $\widetilde{R}=\mathscr{I}(\iota)$, where $\iota=\imath \iota_{0}, \ldots, \iota_{k-1} 2$ is an idempotent of $\widehat{\mathscr{C}}_{\mathscr{C}_{k}}$;

(3) semirings $\widetilde{R}=\mathscr{I} \mathscr{C}(\iota)$, where $\iota=\imath \iota_{0}, \ldots, \iota_{k-1}$ is an idempotent of $\widehat{\mathscr{E}}_{\mathscr{C}_{k}}$;

(4) semiring $\widetilde{R}=\mathscr{R} \mathscr{I}\left(\sigma^{(n)}\left\{a_{0}, \ldots, a_{k-1}\right\}\right)$.

From the last theorem we also find the following.

Corollary 25. There is at least one right identity of the simplex $\sigma^{(n)}\left\{a_{0}, \ldots, a_{k-1}\right\}$. 
If there are two or more right identities of simplex $\sigma^{(n)}\left\{a_{0}, \ldots, a_{k-1}\right\}$, then there is not a left identity. Let us suppose that there is a left identity $\omega \in \sigma^{(n)}\left\{a_{0}, \ldots, a_{k-1}\right\}$. Let $\varepsilon$ be a right identity of the simplex. Then, it follows that $\varepsilon=\omega \cdot \varepsilon=\omega$. If there is a single right identity, from the last theorem, it follows that $a_{i+1}=a_{i}+1$, for any $i=0, \ldots, k-2$. Then, this single right identity is $\varepsilon=\left(a_{0}\right)_{a_{0}+1}\left(a_{0}+1\right) \cdots\left(a_{0}+\right.$ $k-2)\left(a_{0}+k-1\right)_{n-a_{0}-k+1}$. Let $\alpha=\left(a_{0}\right)_{a_{0}}\left(a_{1}\right)_{n-a_{0}}$. We find $\varepsilon \cdot \alpha=\overline{a_{1}} \neq \alpha$, and so we have proved.

Corollary 26. There are not any left identities of the simplex $\sigma^{(n)}\left\{a_{0}, \ldots, a_{k-1}\right\}$.

\section{Conflict of Interests}

The author declares that there is no conflict of interests regarding the publication of this paper.

\section{Acknowledgment}

The author is very much thankful to the Academic Editor for suggestions and support.

\section{References}

[1] J. S. Golan, Semirings and Their Applications, Kluwer Academic, Dordrecht, The Netherlands, 1999.

[2] I. Trendafilov and D. Vladeva, "The endomorphism semiring of a finite chain," Proceedings of the Technical University of Sofia, vol. 61, no. 1, pp. 9-18, 2011.

[3] I. Trendafilov and D. Vladeva, "Endomorphism semirings without zero of a finite chain," Proceedings of the Technical University of Sofia, vol. 61, no. 2, pp. 9-18, 2011.

[4] I. Trendafilov and D. Vladeva, "Nilpotent elements of the endomorphism semiring of a finite chain and Catalan numbers," in Proceedings of the 42nd Spring Conference of the Union of Bulgarian Mathematicians, pp. 265-271, Borovets, Bulgaria, April 2013.

[5] I. Trendafilov and D. Vladeva, "Idempotent elements of the endomorphism semiring of a finite chain," ISRN Algebra, vol. 2013, Article ID 120231, 9 pages, 2013.

[6] W. Bruns and J. Herzog, Cohen-Macauley Rings, Cambridge University Press, 1998.

[7] D. Kozlov, Combinatorial Algebraic Topology, vol. 21 of Algorithms and Computation in Mathematics, Springer, Berlin, Germany, 2008.

[8] I. Trendafilov, "A partition of geometrical structures of the endomorphism semiring," Comptes Rendus de l'Académie Bulgare des Sciences, vol. 66, no. 12, pp. 1661-11668, 2013. 


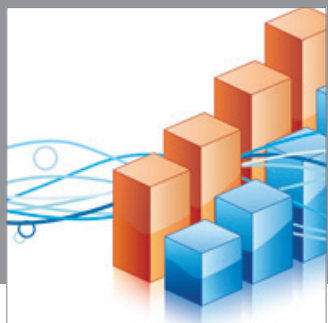

Advances in

Operations Research

mansans

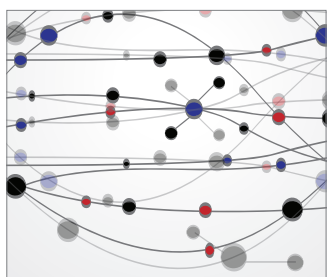

The Scientific World Journal
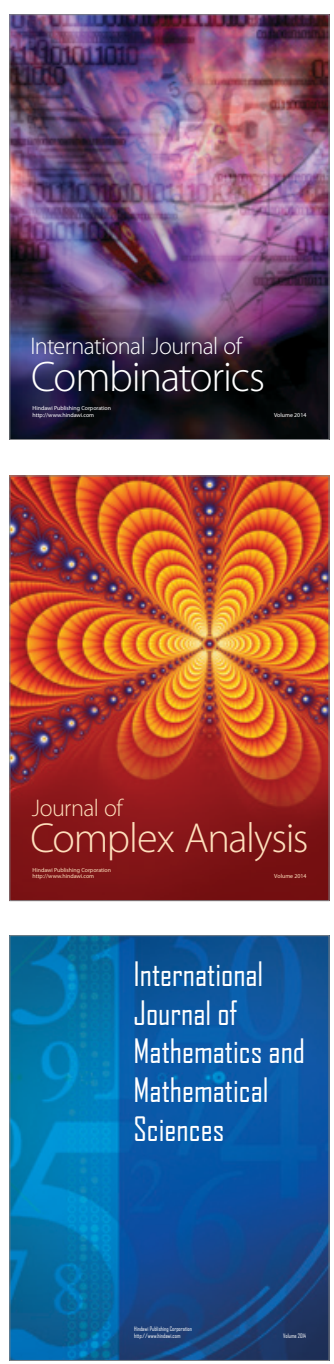
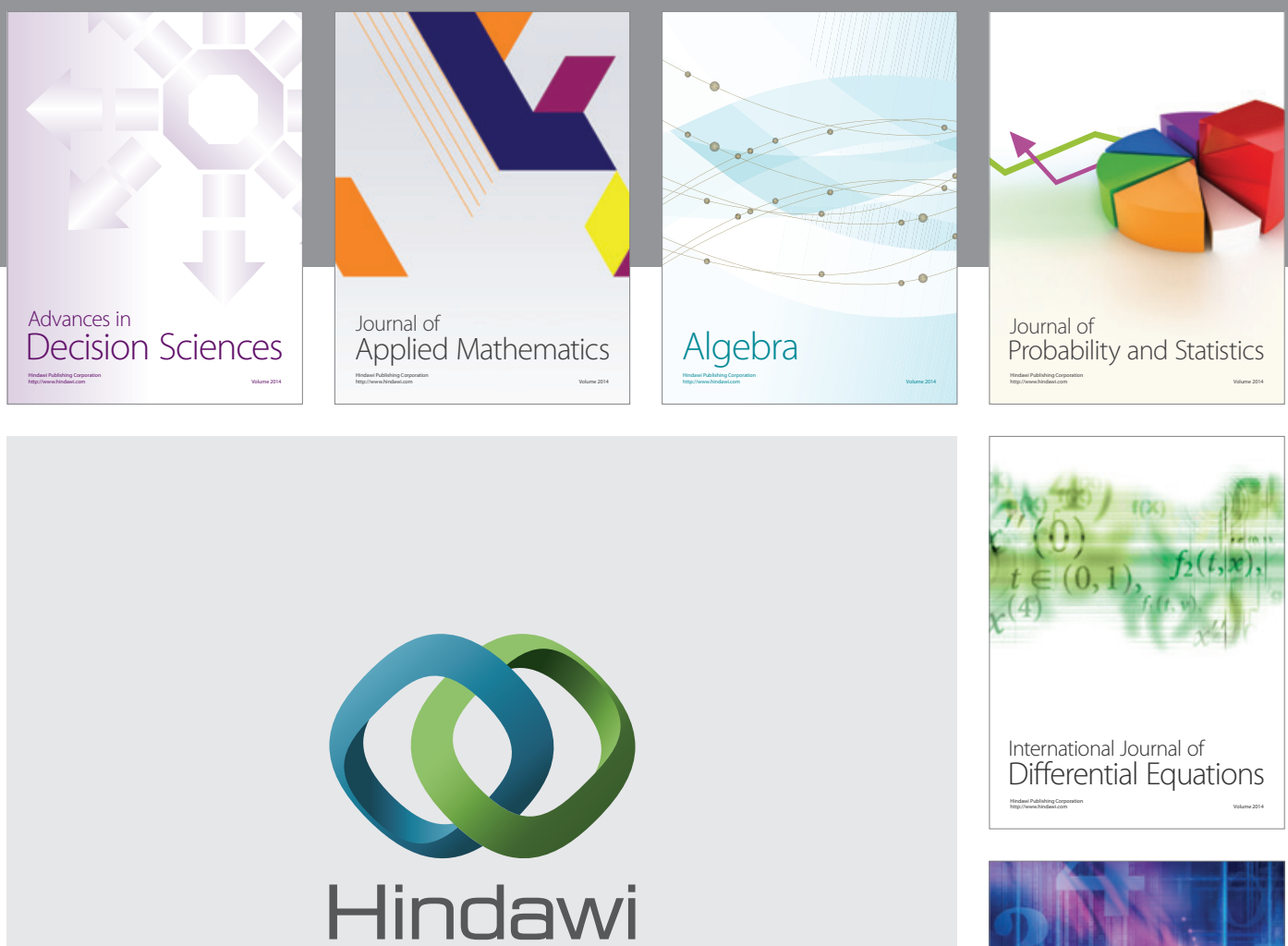

Submit your manuscripts at http://www.hindawi.com
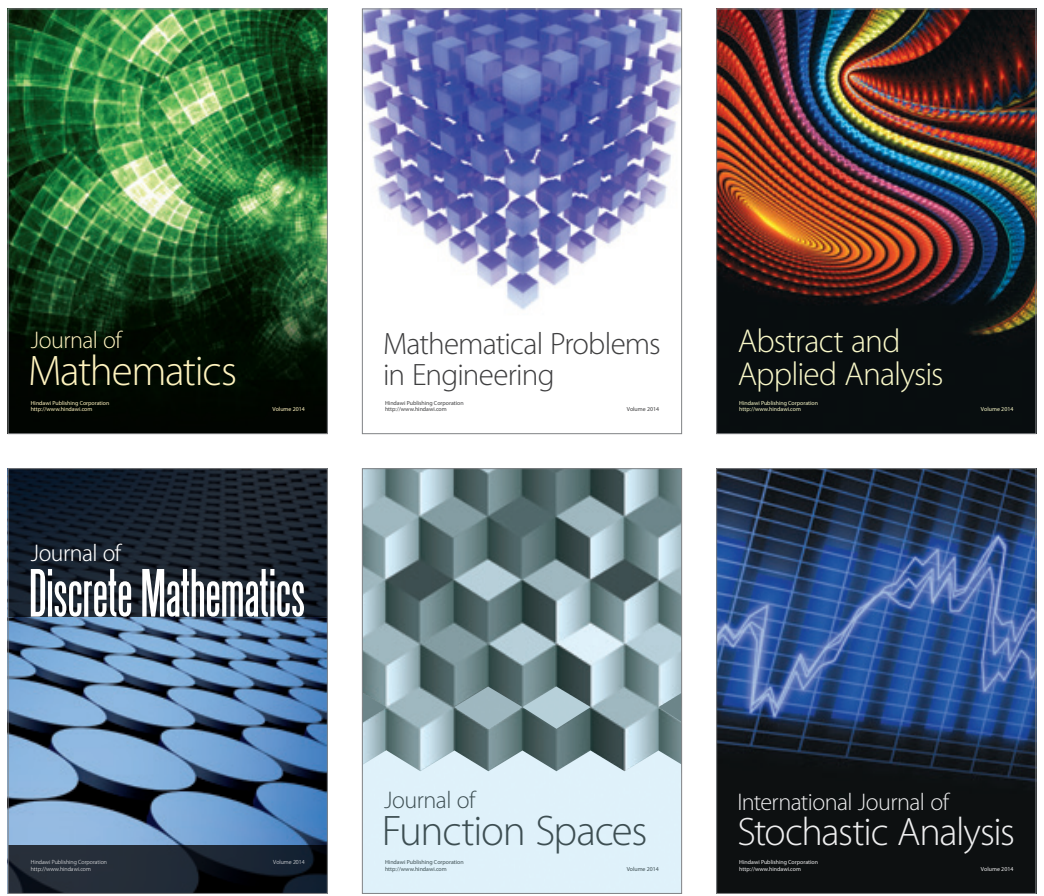

Journal of

Function Spaces

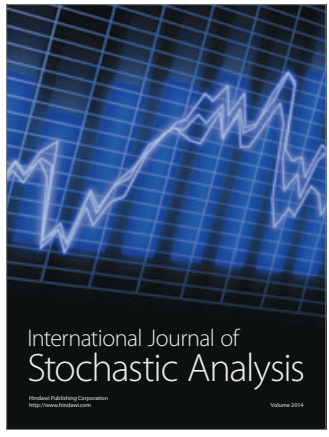

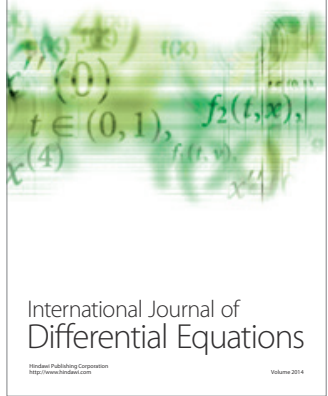
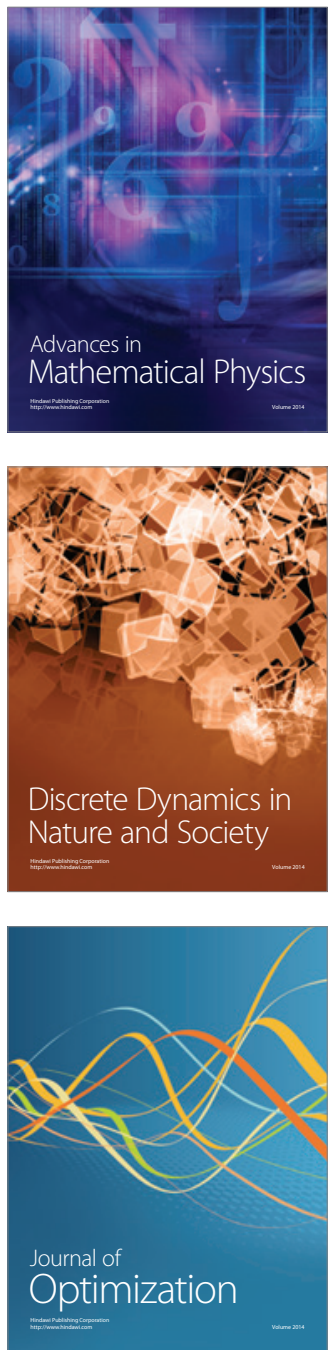